\title{
Verhandlungen der gynäkologischen Section des VIII. internationalen medicinischen Congresses in Kopenhagen, 10.-16. August 1884.
}

\author{
Referat von
}

Dr. S ä $\mathbf{n} \mathbf{g} \mathbf{e} \mathbf{r}$,

Sitzung vom 11. August Vormittags.

Vorsitzender: Stadfeld (Kopenhagen), später Hegar (Freiburg $\mathrm{i} / \mathrm{B}$.$) .$

1) P. Müller (Bern): Der Kaiserschnitt und seine Modificationen.

Der Kaiserschnitt wurde früher als eine weit weniger gefährliche Operation angesehen, als sie in Wirklichkeit ist. Das ergiebt sich aus ihren traurigen Resultaten nicht blos in Anstalten, sondern auch in der Privatpraxis (vgl. die Württemberger Statistik), Von 5 Fällen gingen früher sicher 4 zu Grunde. Dank den Umgestaltungen der Unterleibschirurgie ist jetzt schon eine Besserung der Sachlage erzielt worden und streiten 2 Verfahren um den Vorrang, welche man als die radicale und die conservative Methode bezeichnen kann. Die zu bekämpfenden Gefahren sind vor Allem Blutung und Sepsis. Es ist abzuwägen, welche von beiden Methoden diese am sichersten ausschliesst. Den Punkt der Berechtigung der radicalen Methode, i. e. der Porro-Operation, will Vortr. nicht berühren.

1) Die Blutung (bei der Incision und post partum) lässt sich durch das radicale Verfahren besser beherrschen.

2) Die Sepsis, wie sie ante und intra partum et operationem auftritt, ist bei beiden Methoden gleich gefährlich, doch wird die Gefahr durch Zurücklassung des Uterus noch gesteigert, während 
andererseits, bei der Porro-Operation, gerade die Theile nicht entfernt werden, von welchen am häufigsten die Infection ausgeht, unteres Uterussegment, Collum und Scheide. Hier wären deshalb Modificationen angebracht, wie vollständige Wegnahme des Collum, Umstülpung nach der Scheide u. dgl. In prophylaktischer Beziehung wäre das Beste die frühzeitige Operation.

Bei der conservativen Methode ist die Ausbreitung der Sepsis leichter, ihre Bekämpfung schwerer. $\mathrm{Ob}$ man die eine oder andere Methode wählen soll, ist noch nicht spruchreif, auoh deshalb, weil die neuere Statistik der conservativen Methode noch immer keine sehr reichhaltige ist. Mit Hülfe Sänger's, welcher sich die Förderung der conservativen Methode angelegen sein lässt, hat Vortragender nur ca. 40 Fälle zusammenstellen können mit ca. $40 \%$ Mortalität, denen etwa 150 Fälle von Porro-Operation gegenüberstehen mit ca. $54 \%$ Mortalität.

Wir müssen noch weitere Resultate abwarten. Nach der bisherigen kleinen Statistik bringt die conservative Methode allerdings mehr Fälle durch, aber sie muss, um obzusiegen, noch mehr leisten. Da viele Frauen nach Ueberstehung des Kaiserschnittes nach conservativer Methode später an Ruptura uteri zu Grunde gingen, so muss auch diese noch verhütet werden.

2) Eustache (Lille): Parallele zwischen Embryotomie, Kaiserschnitt und Porro-Operation in Fällen von Beckenenge.

Bei Beckenenge unter $7 \mathrm{~cm}$ Conjugata vera ist nicht die Embryotomie, sondern der Kaiserschnitt auszuführen, nicht die Porro-Operation. Unter 140 Porro-Operationen starben 78 Mütter, 62 kamen davon $=55,7 \%$ Mortalität. Nicht alle von der Operation Geheilten blieben aber gesund. Daucourt ${ }^{1}$ ) fand unter 30 Fällen, die er auf ihr späteres Befinden prüfen konnte, 9 Mal einen von der Operation herrührenden mangelhaften Gesundheitszustand. Man muss sich aber gerade an die definitiven Resultate halten. Dank besserer Hämostase und der Antisepsis hat auch der Kaiserschnitt in den letzten acht Jahren günstigere Erfolge aufzuweisen. E. stellte 45 Fälle zusammen mit 24 Heilungen, gleich einer Mortalität von nur $43 \%$. Sein Freund Dr. Clos madeuc (de Vannes) hat 3 Fälle mit 3 Heilungen aufzuweisen und dies ohne die Vervollkommnungsmittel der neueren Chirurgie. E. hat

1) Resultats cliniques, éloignés des opérations césariennes et de Porro, Paris, O. Berthier. 1884. 
selbst in 3 Fällen assistirt, welche 1880/81 im Departement du Nord vorkamen : 2 wurden geheilt, der 3., wobei der Uterus genäht wurde, starb. - Die Sectio caesarea ist eine einfachere Operation, sie verstümmelt die Frauen nicht und gewährleistet ihnen für später einen besseren Gesundheitszustand als die PorroOperation.

Schlüsse: 1) Der Kaiserschnitt ist der Porro-Operation vorzuziehen. Diese sei nur Ausnahmeoperation. 2) Die moderne Geburtshülfe hat den Kaiserschnitt zu verbessern, um dessen Sicherheit $\mathrm{zu}$ erhöhen. Antisepsis und Uterusnaht sollen allgemein angewandt werden, ebenso wie die provisorische Ligatur des Collum uteri.

\section{Discussion.}

Sänger (Leipzig) setzt die in seinen verschiedenen Veröffentlichungen niedergelegte Kaiserschnittmethode des Näheren auseinander. Sie beruht auf Uebertragung solcher Technicismen, welche der Laparatomie-Technik entnommen auf den Kaiserschnitt anwendbar sind, auf einer angepassten Antisepsis, auf einem den anatomischen und physiologischen Verhältnissen des Uterus angemessenen Nahtverfahren, dessen Hauptprincipien sind: tiefe und breite musculo-musculäre Nähte angelegt unter Vermeidung der Uterushöhle, zahlreiche sero-seröse oberflächliche Nähte mit nicht resorbirbarem antiseptischem Material (Silber, Seide), nach Resection von Streifen der Muscularis in Fällen, wo die Uteruswunde rinnenförmig gestaltet ist und klafft. Auch bei Uteroparietalnaht, die in neuerer Zeit nicht wieder geprüft worden ist, soll die Uteruswunde so geschlossen werden. Eventuell kann die Bauchhöhle durch Jodoformgazewieken drainirt werden analog den Sindons der früheren Zeit. Aseptische Verhältnisse vorausgesetzt ist aber jede Drainage überflüssig. Nach seiner Methode sind bis jetzt 4 Fälle operirt worden: 3 Fälle von Leopold (sämmtlich wegen Beckenenge), welcher überhaupt der Erste war, der die Methode adoptirte; sie heilten sämmtlich. Der 4 . Fall von Beumer (wegen Myoma colli uteri) endete letal an Pyelonephritis: die Uteruswunde fand sich per primam verklebt. S. ist überzeugt, dass seine Methode die Blutung völlig zu beherrschen gestattet und, wofern sie antiseptisch ausgeführt werden kann, eine Primärheilung der Uteruswunde ohne Verdünnung der Narbe gewährleistet. Bestehende Infectionszustände sind nach den für den unverletzten Uterus gültigen Normen zu behandeln. 
Keineswegs verwirft S. die Porro-Operation gänzlich, hat er dieselbe doch selbst $2 \mathrm{Mal}$ ausgeführt; $1 \mathrm{Mal}$ bei Schwangerschaft im rudimentären Nebenhorn eines Uterus bicornis, Heilung; 1 Mal bei Missed labour und multiplen Myomen des Corpus uteri, Tod an Septichämie. Die von ihm für die Porro-Operation aufgestellten 5 Indicationen (s. Monographie S. 199), denen A. Martin als 6. schwere von dem puerperalen Uterus ausgehende Allgemeinstörungen zufügte, hält er aufrecht. Ist nur gegen die allgemeine Anwendung der Porro-Operation, besonders bei Myoma colli uteri, bei Beckenenge und sonst normalem Uterus: hier ist die Amputatio utero-ovarica ungerechtfertigt und nur die conservative Methode am Platz, namentlich wenn die Operation frühzeitig vorgenommen werden kann. Bezüglich der Statistik kann S. der von P. Müller getroffenen Art der Verwerthung en bloc nicht zustimmen, da die Fälle hinsichtlich ihrer technischen Ausführung ungemein differiren. Ebenso wie Clement Godson die PorroOperationen in verschiedene Gruppen gebracht hat, so möchte er dasselbe für die Kaiserschnittfälle thun, in folgender Weise:

I. Fälle nach Sänger's Methode: 4, 3 Heilungen, 1 Todesfall.

II. Fälle aus den letzten Jahren, welche $z$. Th. bei nicht antiseptischer Ausführung sich den von S. vertretenen Principien einer rationellen Uteruswundnaht annähern: 9 Fälle, 6 Heilungen, 3 Todesfälle.

I. und II. zusammen: 13 Fälle,

9 Heilungen $=69,2 \%$.

4 Todesfälle $=30,8 \%$.

III. Antiseptische Kaiserschnitte mit Behandlung der Uteruswunde ohne besonderes Princip: 7 Fälle, 1 Heilung, 6 Todesfälle.

IV. Primitiv àusgeführte Kaiserschnitte (ohne Antisepsis, ohne Uterusnaht): 11 Fälle, 6 Heilungen, 5 Todesfälle.

Die 6 Heilungen sind sämmtlich einer Statistik von Daucourt (1. c.) entnommen, aus den Jahren 1876-1883. Derselbe bringt noch weitere 13 Heilungsfälle aus der Praxis französischer Aerzte zurück bis 1870 .

III. und IV. zusammen: 18 Fälle,

7 Heilungen $=38,9 \%$,

11 Todesfällo $=61,1 \%$.

I. und IV. zusammen: 31 Fälle,

16 Heilungen $=51,6 \%$,

15 Todesfälle $=48,4 \%$. 
Es ergiebt sich also, dass selbst die Gesammtmortalität bemerkenswerth niedriger ist als bei der Porro-Operation.

Leop old (Dresden) macht nähere Angaben über seine glücklichen Kaiserschnitte (im 1. und 3. Fall wurde glatte Heilung erzielt, der zweite Fall war durch ein intraligamentäres Hämatom complicirt); würde künftig von der Resection der Muscularis absehen und nur symperitoneal nähen; hebt die Vortheile der Frühoperation hervor.

Lazarewitsch (Charkow): Bei der Sänger'schen Resection besteht die Gefahr, dass die Wundränder sich nicht mehr exact schliessen lassen, weil nach der Decidua zu, wo diese aneinander stossen, Gewebe fortgenommen wurde. ${ }^{1}$ ) Die Vereinigung der Serosae müsse sich auch durch tiefe und breite Nähte erreichen lassen.

L. Meyer (Kopenhagen) glaubt, dass auch die Gastro - Elytrotomie oder, wie sie in Amerika genannt werde, ,Thomas - Operation" eine Zukunft habe. Er hat dieselbe $1 \mathrm{Mal}$ an der Leiche einer im VII. Monate verstorbenen Schwangeren versucht und war von der Leichtigkeit der Ausführung sehr überrascht.

Breisky (Prag) schliesst sich Müller in den wichtigsten Punkten an. Die Statistiken sind immer noch zu klein, um die Basis eines endgültigen Urtheiles abzugeben. Constatirt, dass die conservative Methode der Porro-Operation, zu deren Anhängern er zähle, Zugeständnisse macht. Glaubt die grössere Sicherheit auf Seite der radicalen Methode. Er hat $5 \mathrm{Mal}$ operirt und alle כ Mütter geheilt, was kein Zufall sein kann, sondern an der Methode liegen muss. ${ }^{2}$ ) Immerhin dürften die Anhänger der Porro-Operation auch Concessionen an die des conservativen Verfahrens machen und halte auch er die Sänger'sche Methode für die geeignetste bei einzelnen bestimmten Fällen.

P. Müller (als Schlusswort) verweist auf das zweifelhafte Schicksal der Frauen, an denen der Kaiserschnitt nach conserva-

1) Kann ich nicht zugeben. Die Resection entfernt nur sehr wenig Muscularis; Contraction und Retraction des Uterus bringen die Wundränder stets wieder in Contact. Die symperitoneale Naht ist freilich die Hauptsache. Liegt aber eine rinnenförmige Wunde vor mit weit abstehenden Rändern der Serosa, dann kann die blosse Naht deren Retraction nur schwer verhindern, ohne die Resection.

2) Bekanntlich erzielte Metz in der vorantiseptischen Zeit anter 7 Kaiserschnittfällen 6 Heilungen. 
tiver Methode ausgeführt wurde. Die Porro-Operation halte er auch für technisch leichter als die geschilderte complicirte Methode. Statistisch müssten doch alle Fälle von Kaiserschnitt zusammengestellt werden, nicht die günstigeren, die nach Sänger'scher Methode etc. getrennt. Zum Vergleich wünsche er dieser für die Zukunft eine recht grosse Anzahl von weiteren Fällen. -

3) Howitz (Kopenhagen): Ueber diagnostische Schwierigkeiten bei Hypertrophia colli uteri.

Bringt weitere Beiträge zur diagnostischen Würdigung der Hypertrophie des Supravaginaltheiles der Cervix uteri während der Schwangerschaft und bei Geschwülsten des Corpus uteri, Verhältnisse, auf welche zuerst A. Martin die Aufmerksamkeit gelenkt hat (Zeitschr. f. Geburtsk. u. Gynäkol. Bd. VI. S. 101). Greift von den 13 Fällen, die er beobachtete, besonders 4 heraus, 3 bei Schwangerschaft, 1 bei Myoma corporis uteri. Von grösster Bedeutung für die richtige Diagnose ist Hochstand und auffallende Beweglichkeit des Corpus, welche auf Verlängerung des Collum hinweise. In den ersten Monaten der Schwangerschaft namentlich kann man bei solchen Fällen schlimmen Irrthümern ausgesetzt sein, indem das weiche, schwangere Corpus bei der Länge des Collum, welches an der Auflockerung nicht theilnimmt, sondern rigid bleibt, leicht für einen Tumor, namentlich für ein Cystoma ovarii gehalten werden und man in Versuchung kommen könne zu sondiren, oder die Laparatomie zu machen.

A. Martin (Berlin) findet in Howitz's Mittheilungen eine volle Bestätigung dessen, was er von seinen eigenen 7 ersten Fällen dargelegt habe. Es handle sich mehr um eine Elongatio colli uteri als um Hypertrophie. Besonders wichtig ist, dass bei vorhandener Schwangerschaft deren Zeichen sehr wenig ausgeprägt sind, ja dass sogar die Menses andauern können. Glaubt, dass Endometritis cervicis die Ursache abgiebt, wenigstens sind die entsprechenden Krankheitserscheinungen vorhanden.

Priestley (London) hat diese Elongatio colli besonders oft bei Prolapsus vaginae gesehen. Bei Schwangerschaft ist die Diagnose nicht leicht vor dem VI. Monate zu stellen und können die Contractionen des schwangeren Uterus (Braxton-Hicks) hier ein wichtiges Hülfsmittel abgeben. Erzählt einen Geburtsfall bei bestehender Hypertrophie : Forceps, lebendes Kind, gutes Wochenbett. Die Geburt scheint überhaupt durch die Hypertrophia colli nicht gestört zu werden, wenngleich sie dadurch erschwert werde. 
Howitz ist mit A. Martin nur in dem Punkte nicht ganz einig, dass die betreffenden Frauen alle krank sein und bei zugetretener Schwangerschaft weiter menstruiren sollen, was er nicht beobachtet habe. Priestley stimme er darin bei, dass die Hypertrophie nie ein Geburtshinderniss abgebe, weshalb die exspectative Behandlung intra partum das beste sei.

\section{Sitzung vom 11. August Nachmittags.}

Vorsitzender: Pippingsköld (Helsingfors).

4) Koeberlé (Strassburg): Die Behandlung der Fibromyomedes Uterus durch die Laparatomie.

Sowohl die Indicationen zur Operation wie das Verfahren bei derselben sind noch immer Gegenstand von Controversen. Als Indicationen können gelten: 1) übermässige und langwierige Blutungen, sowie rasches und fortschreitendes Wachsthum der Geschwulst; 2) das relative Alter der Patientin, wenn der Eintritt der Menopause noch fernliegt; 3) der Sitz des Tumor (nur im Corpus uteri bei normaler Cervix; nicht im Ligamentum latum; in der Kleinbeckenhöhle bei Bestand von Obstructionserscheinungen); 4) bei sonst unerträglichen Beschwerden (durch Behinderung, Hinfälligkeit, Arbeitsunfähigkeit etc.). Contraindication bilden: ausgedehnte und blutreiche Verwachsungen mit der vorderen Bauchwand und mit Eingeweiden; Einkeilung in der Beckenhöhle; zunehmender und rasch sich ersetzender Ascites; Bestand einer begleitenden unheilbaren Krankheit oder eines Leidens, welches die Heilung ungünstig beeinflussen kann (Syphilis, Hämophilie, Bronchitis chronica etc.); sehr alte, harte, stationäre Tumoren; kleine Geschwülste bei Nulliparen mit straffen Bauchdecken; sehr fette Bauchdecken. In den günstigsten Fällen mit Sitz am. Fundus uteri, welche natürlich die besten Resultate ergeben, ist das Leben selten in Gefahr.

Das Operationsverfahren ist verschieden je nach Sitz und Grösse der Geschwulst. Für die Operation von der Scheide her eignen sich; die fibrösen Polypen der Uterushöhle; breitbasige submucöse Myome, sobald sie in die Scheide hineinragen und die Genitalien weit genug sind, ihre Entfernung zu gestatten. Grössere nur in die Uterushöhle ragende submucöse Myome, interstitielle und subseröse Geschwülste verfallen der Laparatomie. Diese wird 
stets in der Linea alba vorgenommen. Besteht eine Hernia umbilicalis, so wird der Bruchsack mit ausgeschnitten. Das Peritoneum wird nach einer kleinen Incision mit zwei Fingern weit aufgerissen. Die Länge des Bauchschnittes ist gleichgültig, er gehe aber so tief herab als möglich.

Gestielte Geschwülste werden abgetragen nach Bildung einer Schnürrinne mittels Serre-noeud und Seidenligatur. Versenkung. Catgut und Metallligatur sind gefährlich. Kleinere, breitbasige, umschriebene Myome werden ausgeschält; die Blutstillung geschieht durch zahlreiche tiefe und oberflächliche Ligaturen (,ligatures perdues"), ein Verfahren, welches er 1878 zum ersten Male angewandt hat. Bei den grösseren Myomen wird der Uterus mitentfernt: Hysterotomie, „Hysterectomie suspubienne“. Hier bieten wieder die Myome des Corpus günstigere Verhältnisse dar als die des Collum, besonders wenn sie unbeweglich den Douglas und das Becken einnehmen. Ist die Geschwulst fixirt und zwischen den Ligamenta lata entwickelt, dann stets sehr schwierige, ja unausführbare Operation. Die Lig. lata müssen mit ihren Gefüssen durch versenkte Massenligaturen abgebunden werden, wobei die Ovarien mitwegzunehmen sind. Darauf Ablösung der Blase vom Uterus und Umstechung der Uteringefässe. Ist die Totalexstirpation des Uterus nothwendig: quere Eröffnung des vorderen Scheidengewölbes von oben her und Durchziehen der Metalldrähte des Serre-noeud durch die Scheide. Lässt man das untere Stück des Uterus sitzen, werden die Metallligaturen durch das Collum geführt, in 2 Serre-noeuds gefasst und diese an der Bauchwand befestigt. Nach diesem Verfahren - also extraperitoneal - hat K. seit 1853 in etwa 50 Fällen operirt mit einer Mortalität zwischen 5 und $10 \%$.

Die metallische Ligatur und deren Festigung mittels Serrenoeud kann wegen der Gefahr von Nachblutung nicht wohl durch versenkte Suturen ersetzt werden. Ist es nicht möglich, wie bei intraligamentärer Entwickelung der Geschwülste metallische oder auch elastische Ligaturen anzuwenden, dann kann die Enucleation gemacht werden, ein schwerer Eingriff, nach welchem Heilung die Ausnahme bildet. Eventuell ist hier besser die Castration am Platze. Die Schröder'sche Methode, welche allerdings die Nachbehandlung sehr vereinfachen würde, sei in praxi voller Gefahren und sollte die keilförmige Ausschneidung nicht verallgemeinert werden. Das Morcellement Péan's sei unnütz und gefährlich. 
Zum Schlusse schildert K. seine bekannte Bauchnaht. Seit zwei Jahren behandelt er den Stumpf mit Jodoform und Jodoformcharpie. Auch auf die Ligatures perdues wird Jodoform gestäubt, stets in geringer Menge. Der Verband ist häufig zu wechseln, eventuell 2-3 Mal am Tage. Nach dem Abfallen des Stumpfes Collodiumverband.

Olshausen (Halle a/S.) stimmt nicht in allen Punkten mit Koeberlé überein, hält seine Contraindicationen für zu beschränkende. Z. B. sei gerade Einklemmung im Becken eine Indication, weil doch die Möglichkeit bestehe, die Geschwulst herauszuheben. Auch Ascites erkenne er nicht als Contraindication, ebensowenig gefässreiche Adhäsionen : sie lassen sich nicht diagnosticiren und bilden, weil sie das Wachsthum der Geschwulst fördern, eher eine Indication. Schildert einen Fall von Myom des Lig. rotundum mit colossal gefässreichen Adhäsionen, wobei es doch gelang die Geschwulst zu entfernen. Es sind besonders zwei Schwierigkeiten, denen man begegnen kann: 1) subseröse und intraligamentäre Entwickelung derart, dass man nicht an die Basis heran kann: macht man hier seitlich Massenunterbindung, wobei die Spermaticalgefässe mitgefasst werden; öffnet das Peritoneum möglichst tief unten mit einem feinen Bogenschnitt, dann ist die Ausschälung meist leicht und fast blutlos. Man achte aber darauf, den Urether zu vermeiden. Freilich sind auch so nicht alle Fälle operirbar: eventuell kann ein Theil der Geschwulst zurückgelassen werden. - Die zweite Schwierigkeit besteht darin, dass der Tumor nicht blos in den supravaginalen Theil dex Cervix, sondern durch das Os externum bis in die Vagina hinabragt: hier wäre die Totalexstirpation zu machen, doch kann und darf man gut den unteren Theil der Geschwulst sitzen lassen, mit Hülfe der elastischen Ligatur, welche er Koeberlé gegenüber warm vertheidigt. Ihr gehöre die Zukunft. Durch Aufbewahren in Sublimat 1\% (1 Procent!), dann durch Einreiben mit Jodoformglycerin vor dem Gebrauche lassen sie sich vollständig aseptisch halten. O. ist absolut gegen die extraperitoneale Behandlung des Stumpfes und gegen Drainage, schon in Hinblick auf die ungeheure Vereinfachung der Nachbehandlung, ist überzeugt, dass die extraperitoneale Behandlung der intraperitonealen weichen werde.

(Vorläufiger Abbruch der Discussion).

5) Apostoli (Paris): Die elektrische Behandlung der Fibromyomedes Uterus. 
296 Verhandlungen der gynäkolog. Section des VIIJ. internationalen

Gegenüber der relativ unwirksamen medicinischen, gegenüber der chirurgischen Behandlung der Uterusmyome ist die elektrische am einfachsten und ungefährlichsten. Im Gegensatze zu der früheren intravaginalen Elektrisation mit schwachen Strömen wendet er die intrauterine Application starker Ströme an bis $\mathrm{zu} 100$ Milliampère. Ist die Uterushöhle nicht zugängig, so kann man die uterine Elektrode in eine elektrische Nadel verwandeln und mit derselben von der Scheide her eine Elektropunktur ausfïhren. Die im Uterus befindliche negative Elektrode soll eine cauterisirende, die breite, biegsame auf die Bauchhaut gesetzte, positive Elektrode soll eine ,zertheilende“ Wirkung ausüben. Die Cauterisationen hinterlassen keine Narbe. Anwendung in über 100 Fällen, zwar nicht mit völligem Schwinden der Geschwulst, doch mit stetiger Verkleinerung des Uterus und Beseitigung der Beschwerden.

6) Wiedow (Freiburg i/B.): Castration bei Uterusfibromen.

Dankt für das ihm von allen Seiten reichlich zugeflossene Material, welches ihn in den Stand setzt über 112 einschlägliche Fälle zu berichten. Von denselben sind 17 gestorben, meist an Sepsis. Betreffis des Einflusses der Operation auf die Blutungen und das Wachsthum der Geschwülste, bekam er über 73 Fälle Auskunft. In 53 derselben trat vollständige Menopause ein, in 36 gleichzeitig Abnahme des Tumor etc. (Detaillirte Zahlenangaben s. in den Comptes rendus des Congresses I).

Zur Illustration der Einwirkung der Castration auf Myome des Collum werden zwei Fälle genauer geschildert: in dem einen Falle (Np.) trat Aufhören der Blutung und Abnahme der Geschwulst ein (Hegar), in dem anderen bei einer 40jährigen VIp. mit ständiger Incontinentia urinae, als deren Ursache ein Myom der vorderen Collumwand erkannt wurde, erfolgte Sistiren der Blutung und konnte der Harn zurückgehalten werden, obwohl die Geschwulst nicht kleiner wurde.

Die Grösse der Geschwulst ist keine Contraindication gegen die Castration.

Da die Mortalität der Myomotomie ca. $33 \%$ beträgt, die der Castration aber weit niedriger ist, so sollte diese bevorzugt und die Myomotomie nur auf fibro-cystische Geschwülste und gut gestielte subseröse Myome beschränkt werden. Jedenfalls soll man bei jeder Myomotomie die Ovarien mit entfernen.

W. widerlegt schliesslich die Ansicht von Lawson Tait von 
der Bedeutung der Tuben auf die Menstruation als eine irrige. Hegar, Tauffer, Gusserow u. A. sahen Menopause und Geschwulstabnahme, trotzdem die Tuben zurückblieben und ungekehrt Wachsthum des Tumor, wenn die Tuben weggenommen wurden. Die sogenannte Lawson Tait'sche Operation sei nichts anderes als die Hegar'sche Castration.

In den Comptes rendus des Congresses werden veröffentlicht werden die angekündigten, doch nicht gehaltenen Vorträge von:

P. Ménière (Paris): ,Zerstörung der Fibromyome der Gebärmutter durch elektrolytische Punctur" und

Margary (Turin): „Amputatio supravaginalis uteri von der Scheide aus bei Fibromyomen der hinteren Gebärmutterwand."

\section{Sitzung vom 12. August Vormittags.}

Vorsitzender: Priestley (London).

Fortsetzung der Discussion über Myomotomie:

Sir Spencer-Wells (London) stellt sich auf Olshausen's Seite, was die Erweiterung der von Koeberlé bezeichneten Contraindicationen anlangt. Die Gefahren der Operation lassen. sich durch die auf Scheide und Uterus ausgedehnte Antisepsis (Jodoform!) sehr mindern. Die Erfolge der extraperitonealen Stumpfbehandlung sind auch in England sehr gute, doch waren sie es seinerzeit auch, als noch die Klammerbehandlung bei der Ovariotomie üblich war. Er glaubt an die Zukunft der intraperitonealen Methode, namentlich mit Verwendung der elastischen Ligatur. - Vertheidigt gegen Koeberlé, welcher das Peritoneum parietale nicht mitfasst, weil es sich von selbst vereinige, seine eigene Methode der Bauchnaht. Auch hinsichtlich der Drainage schliesst er sich Olshausen an. Er drainirt fast gar nicht mehr. Ueber die Wahl zwischen Castration und Myomotomie lasse sich jetzt noch keine Entscheidung treffen.

Hegar (Freiburg i/B.) hält es nicht für angemessen, einen so strengen Gegensatz zwischen intra- und extraperitonealer Methode zu machen, da die entschiedensten Anhänger der letzteren gelegentlich auch die erstere wählen z. B. bei gestielten, subserösen Tumoren, oder wenn der Hals der Geschwulst sehr schmal ist, oder wenn diese leicht auszuschälen geht. Für andere Fälle 
möchte er aber doch der intraperitonealen Methode keine grosse Zukunft prognosticiren; die Schröder'sche Methode sichert eben nicht gegen Blutung. Ein grosser Fortschritt wäre hier gewonnen, wenn stets mit elastischer Ligatur operirt werden könnte. Ihm sind die Versuche nicht gelungen: er bekam wiederholt intraabdominelle Abscesse.

Die extraperitoneale Methode bietet am meisten Sicherheit gegen Blutung und Sepsis. Für den Operateur ist sie ja nicht bequem, aber sie ist sicherer. Die Stielversorgung bei der Ovariotomie kann nicht zum Vergleich herangezogen werden, da die Verhältnisse hier ganz andere sind. Eine Prophezeiung für die Zukunft bat stets etwas Missliches!

Als ,wilde" Operationen möchte $\mathrm{H}$. die der subperitonealen und der Collum-Myome bezeichnen. Wohl hat Spencer-Wells ein Mal ein colossales Myom ausgeschält und dabei nur ein Gefäss unterbunden, ebenso er selbst einen ähnlichen Fall, in einem anderen hat $H$. aber die Vena epigastrica durchschnitten und die Patientin an Nachblutung verloren. Sehr schwer ist auch zu bestimmen, wie der Urether mit dem subperitonealen Myom verbunden ist. Nur wenige Fälle von Enucleation bei Collummyomen von der Bauchhöhle aus sind günstig abgelaufen. Gerade für diese Fälle empfehle er mit Wiedow die viel leichtere Castration.

Knowsley Thornton (London) unterscheidet 1) solche Fälle, die gar nicht operirt werden sollten; 2) solche, die nicht operirt werden können; 3) solche, welche operirbar sind durch Hysterotomie (Mitentfernung nur eines Stuickes Uterus), Castration, Enucleation, Hysterectomie (Entfernung des grössten Theiles des Uterus oder des ganzen Organs). Bei Hysterotomie sah er aus ganz dünnen Stielen enorme Blutung. Die Castration hält er mehr für kleine als für grosse Geschwülste geeignet. Wie Olshausen hält auch er Koeberlé's Contraindicationen für zu weitgehend. Dagegen spricht er sich für die extraperitoneale Stumpfbehandlung Ko e ber lé's mit Serre-noeuds aus. Der grösste Stumpf könne durch solche ungemein verkleinert werden. Vor der Anlegung sollen die Ligamenta lata für sich unterbunden werden.

Martin (Berlin) hat den Ausführungen von Olshausen und Spencer-Wells nur wenig hinzuzufügen. Bei seinen etwa 50 betragenden Lapara-Myomotomien suchte or stets die ganze Geschwulst zu entfernen. Bei Collum-Myomen verfuhr er ähnlich wie Schröder. Er erklärt sich gegen die extraperitoneale Methode und hält es für das Idealste, den Stumpf mit Peritoneum zu 
decken. Statt der Spencer-Wells'schen Klammern wendet er für dünne Stümpfe nur geflochtene Seide an, sonst bis zur Vollendung der Naht die provisorische elastische Ligatur. Seit zwei Jahren macht er auch stets die Drainage durch den Douglas.

Howitz (Kopenhagen) hat 13 Fälle operirt, 3 Mal mit Eröffnung der Uterushöhle. 5 Todesfälle, meist an Sepsis. Stets extraperitoneale Behandlung. Hält die Operation nur in den extremsten Fällen für zulässig, da die Gesammtmortalität noch immer gegen $40 \%$ betrage. Auch Keith erklärte die Operation als ultimum refugium. Die Blutungen beruhen auf eigenthümlichen, von Kampe beschriebenen Veränderungen der Mucosa uteri und der Ovarien. Ein indifferenteres Verfahren (besonders die Abrasio mucosae) ist vorzuziehen.

Kaltenbach (Giessen): Wenn die Indication zum Operiren dringend ist, dann liegt der Fall gewöhnlich ungünstig und umgekehrt. Er vermisst bei Gestellung der Indication eine Anzahl von Gefahren wie Thrombose der Beckenvenen, Embolieen, Phlegmasia alba, Decubitus, Zerfall der Geschwulst etc. Was stark gefässhaltige, flächenhafte parietale Adhäsionen anlangt als Contraindication, so sind sie selten, aber sie führen zu rascherem Wachsthum der Geschwulst, sie „füttern" dieselbe und machen durch Zerrung sehr heftige Beschwerden. Er operirte einen solchen Fall mit Erfolg, wo ein anderer Operateur davon abgestanden war. Er huldigt der extraperitonealen Methode. Von seinen 10 zuerst veröffentlichten Fällen starb 1, von 5 weiteren keiner, dagegen verlor er 2 nach Schröder intraperitoneal behandelte Fälle.

Sänger (Leipzig) spricht sich zu Gunsten der intraperitonealen Methode aus und bedient sich eines Verfahrens, welches als Combination desjenigen von 0lshausen und Schröder gelten kann. Ohne vorherige Durchtrennung der Ligamenta lata wird eine $3 \mathrm{~mm}$ starke Gummischnur sogleich als Dauerligatur angelegt and nach Thiersch mittels Bleiring festgeklemmt. Nach geschehener Amputation wird die Mucosa der Uterushöhle ausgeschnitten, der Trichter mittels Thermokauter gebrannt, mit Chlorzink ausgetupft und mit Jodoform gefiullt. Die Ligamenta lata werden noch besonders durch Seidenligaturen zusammengerafft und der Stumpf derart vernäht, dass das lang vorstehen gelassene Peritoneum eingefalzt wird und breit-flächenhaft, nicht wie nach Schröder nur lineär, aneinanderliegt. Darauf Versenkung des dergestalt symperitoneal vernähten Stumpfes mitsammt 
Gummischnur und Bleiring. Von den so behandelten 4 Fällen heilten 3 ohne Reaction. Im 4. Fall stiess sich einige Wochen später das Schnürstïick durch die Cervix ab (dünner Stumpf, zu frühe Untersuchung in der Klinik, Exsudatbildung). Jetzt ist die Frau gesund. S. legt grosses Gewicht darauf, die elastische Ligatur nicht so fest anzuziehen, dass der Stumpf völlig anämisch wird: er soll nicht ausser Ernährung gesetzt werden. Die mitversenkte elastische Ligatur schützt vor Nachblutung, die Desinfection von Scheide und Collum vor und während der Operation sichert vor Infection des Stumpfes, die symperitoneale Naht vor Infection der Bauchhöhle. S. kann daher Hega r's Bedenken gegen Versenkung elastischer Ligaturen nicht theilen. $\mathrm{Zu}$ den von Kaltenbach angeführten bedrohlichen Complicationen, welche u. A. frühzeitiges Eingreifen erheischen, möchte S. mit Ros e noch die Herzdilatation rechnen. Er verlor einen Fall von primärem Myom des Ligamentum latum an solcher 12 Stunden nach der keineswegs sehr blutigen Operation.

Koeberlé (zum Schlusswort): Er hat wiederholt bei Bestand von Ascites operirt und öfter Todesfälle erlebt, oder auch Wiederkehr des Ascites trotz Operation. Hält im Allgemeinen seine Contraindicationen aufrecht, gesteht aber ausnahmsweise Nichtberücksichtigung derselben zu. Schiebt ein gut Theil seiner günstigen Resultate auf den Gebrauch von Servietten statt von Schwämmen. Bedauert sehr Spencer-Wells' Methode der Bauchnaht nicht annehmen zu können. Den Vergleich mit der Ovariotomie findet er nicht zutreffend: von 100 Fällen mit extraperitonealer Behandlung des Stieles verlor er 11, von $60 \mathrm{mit}$ intraperitonealer nur 4. Für die Myomoperationen ist z. Z. die extraperitoneale Behandlung die beste. Ueberdeckung des Stumpfes mit Peritoneum hält er, wenn intraperitoneal verfabren werde, für unnöthig.

7) Miculicz (Krakau): Antiseptik bei der Laparatomie.

Nach einleitenden Bemerkungen über die Umgestaltungen und Fortschritte der Laparatomie durch die Antiseptik, über die Operateure, welche sie, wie Laws on Tait, für unnöthig erklären, dabei aber doch sich einer nur unvollkommenen Antiseptik bedienen, constatirt er eine so gut wie allgemeine Uebereinstimmung im Princip der Sache, Differenzen nur noch in Einzelheiten, beruhend auf den Eigenthümlichkeiten des Peritoneum, welche die Aufgaben der Antisepsis zum Theil erleichtern, zum Theil wesentlich erschweren. Wenn antiseptische Vorkehrungen, 
Drainage und Occlusivverband die drei Pfeiler der Lister'schen Methode ausmachen, so sind die letzten beiden für die Laparatomie von minderer Wichtigkeit, meist entbehrlich; andererseits bieten die grosse Flächenausdehnung des Peritoneum, seine hochgradige Resorptions- und Transsudationsfähigkeit wieder erschwerende Momente für die Technik hinsichtlich der Ableitung und Unschädlichmachung der Secrete, welcher allerdings wiederum normale Beschaffenheit des Bauchfelles, sowie die Fähigkeit rasch plastische, einkapselnde Exsudate zu liefern, günstig entgegenkommt. Da nun das Peritoneum gegen septische Infectionsstoffe empfindlicher ist als alle anderen Gewebe, eine Irrigation der Bauchhöhle mit stärkeren Antiseptica unmöglich, die Drainage fast wirkungslos ist, so liegt die Hauptaufgabe der Antisepsis in der absoluten Abhaltung von septischen Stoffen, also in der Prophylaxe. Bei aseptisch ausgeführten Operationen ist die Drainage in den allermeisten Fällen ganz überflïssig, ja wegen der Möglichkeit nachträglicher Infection gefährlich und aus mechanischen Gründen in ausreichender Weise undurchführbar. Immer indicirt ist sie nur bei abgegrenzten Exsudaten. Die zweite Hauptaufgabe der Antisepsis bei Laparatomien ist die Vermeidung von „todten Räumen“, das heisst: Ansammlung von zersetzungsfähigem Secret in der Bauchhöhle. Ihr wird genügt durch genaueste Toilette der Peritonealhöhle, wie sie von Koeberlé eingeführt wurde; Vermeidung secernirender Wundflächen durch Massenligatur, Abbrennen von Stiel und Adhäsionen, Vernähung von Defecten z. B. der Ligamenta lata, des Uterusstumpfes, Einnähen von Cystenresten, bez. des Peritonealüberzuges ausgeschälter Geschwülste in die Bauchwunde; Vermeidung von Irritation des Bauchfelles durch stärkere Antiseptica. Das Nahtmaterial ist gleichgültig, wenn es nur aseptisch ist, ebenso, ob man den Stiel extra- oder intraperitoneal versorgt, sobald nur die Bauchhöhle nach aussen vollkommen abgeschlossen bleibt; ebenso die Bauchnaht und der Wundverband, wenn nur die Peritonealflächen vereinigt sind. Doch empfiehlt es sich, zur Beförderung der Resorption einen gleichzeitig fixirenden Compressionsverband anzulegen. - Die Carbolsäure scheint unter den verwendeten antiseptischen Lösungen noch immer die beste und sicherste zu sein. Ueber das Sublimat liegen noch keine genügenden Erfahrungen vor. Das Jodoform findet in kleinerer Menge (nicht über 6,0) vortheilhafte Verwendung zum Bestäuben des Stiels oder des vernähten Uterusstumpfes. 
302 Verhandlungen der gynälolog. Section des VIII. internationalen

Küstner (Jena) lenkt die Aufmerksamkeit auf das prophylaktisch-aseptische Verfahren der Desinfection von Operationsräumen durch Bromdämpfe, welche nach $\mathrm{K}_{0 \mathrm{ch}}$ hierzu am wirksamsten sein sollen. Man bediene sich hierzu der von der Schering'schen Apotheke in Berlin gelieferten Brom-Silicatstangen, um binnen wenigen Stunden alle im geschlossenen Raume befindlichen Bacterien zu tödten, alle Gerüche zu beseitigen.

Olshausen (Halle a/S.) perhorrescirt das Einnähen von Cystenresten in die Bauchwunde vollständig, weil es stets fatale Eiterung, im besten Falle noch eine Fistel hinterlasse. Räth den Cystenrest stets zu versenken, auch wenn er noch so ,unreinlich“" aussieht. Die Erfolge dieses Verfahrens sind merkwürdig günstige, sogar bei papillaren Cystomen, die intraligamentär und subperitoneal entwickelt oft bis zum Anus hinabreichen und nicht vollständig exstirpirt werden können.

Mic u licz (Krakau): Missverständniss! Er meinte nicht eigentlich den Cystenrest, sondern die peritoneale Hülle der Cyste, des Tumor.

8) Simpson (Edinburgh): Ueber eine gemeinsame geburtshülfliche Nomenclatur.

Entwickelt nochmals die von ihm auf dem internationalen Congress in London aufgestellten Normen und fragt nach den Ergebnissen der Thätigkeit der für die verschiedenen Länder damals ernannten Commissionsmitglieder ( $\mathrm{Hen}_{\mathrm{n}} \mathrm{ig}^{\mathrm{l}}$ ) für Deutschland, Budin für Frankreich, Halbertsma für Holland, Stadfeldt für die scandinavischen Länder, Lazarewitsch für Russland, Mangiagalli für Italien, King für Amerika).

Als Beispiel der Einfachheit und Folgerichtigkeit der Nomenclatur giebt er unter Anderem das englische Schema der Lagen und Stellungen:

Praesentations. Positions.

$\left\{\begin{array}{clll}\text { Vertex } \\ \text { Face } \\ \text { Pelvis } \\ \text { Shoulder }\end{array} \quad \begin{array}{lll}\text { Left } & \begin{array}{l}\text { Occipito } \\ \text { Mento }\end{array} & \text { anterior } \\ \text { Sacro } & \text { posterior } \\ \text { Acromio } & \end{array}\right.$

Die Uebertragung in andere Sprachen unterliege wohl keiner Schwierigkeit. -

1) S. dessen Bericht in den Verhandl. d. Gesellsch. f. Geburtsh. z. Leipzig, Sitzg. v. 21. Novbr. 1881. Centralbl. f. Gynäk. 1882, No. 8. 
Priestley's (London) Antrag, die Commission fiir den nächsten Congress bestehen zu lassen, da deren Arbeiten noch nicht abgeschlossen seien, wird angenommen.

\section{Sitzung vom 12. August Nachmittags.}

Vorsitzender: Olshausen (Halle a/S.).

9) Halbertsma (Utrecht): Die Bedeutung der Albuminuria gravidarum. (S. die Abhandlung des Vortragenden in Nr. 212 von Volkmann's Sammlung klinischer Vorträge.) Die Albuminurie der Schwangeren kann abhängen von irgend einer auch bei Nichtschwangeren vorkommenden Erkrankung oder sie ist der Schwangerschaft eigenthümlich als eine Begleiterscheinung der Nephritis gravidarum. Der ätiologische Zusammenhang derselben mit der Schwangerschaft ergiebt sich aus dem allmäligen Einsetzen, der raschen Zunahme mit fortschreitendem Wachsthum des Uterus, der Abnahme der Urinmenge und dann der plötzlichen Besserung nach Entleerung des Uterus. Wie entsteht nun die Albuminuria gravidarum? Die von Cohnheim, Spiegelberg und Fleischl vertretene Theorie einer reflectorischen $\mathrm{Zu}-$ sammenziehung der Arteriae renales, also einer reflectorischen Anämie der Nieren, ist eine Hypothese, die deshalb wenig für sich hat, weil diese Zusammenziehung nicht wohl eine dauernde sein kann, sodann weil die Blutgefässe der Niere vom schwangeren Uterus gar nicht behindert werden. Wohl aber liegt dieser zwischen den Uretheren und können dieselben nicht blos direct seitens des Uterus comprimirt werden, sondern dies kann auch geschehen, wenn zwischen der Grösse des schwangeren Organes und der Bauchhöhle ein Missverhältniss besteht, so dass in dieser ein erhöhter Druck zur Geltung kommt. Daher das häufigere Auftreten von Albuminurie und Eklampsie bei Erstgeschwängerten, bei Zwillingsschwangersehaft. So hat man auch nach dem Tode des Fötus Aufhören der Albuminurie und Eklampsie beobachtet wegen eingetretener Minderung in der Spannung der Bauchdecken. In den meisten Fällen ist die Albuminuria gravidarum somit bedingt durch die Compression und Dilatation der Harnleiter, welche dann durch sich alsbald anschliessende Lähmung noch weiter ausgedehnt werden. Diese Uretherendilatation bei Eklampsie mit Albuminurie ist auch von Anderen gefunden worden, z. B. von Löhlein 
in 32 Fällen $8 \mathrm{Mal}$. Doch findet man sie deshalb nicht immer, weil sie post partum rasch zurückgehen kann. Eine weitere Stütze findet H.'s Annahme von der Entstehung der Albuminuria gravidarum auch in den Experimenten von Aufrecht, welcher nach Unterbindung der Uretheren Nephritis auftreten sah. Es stimmen also anatomischer und klinischer Befund, sowie das Thierexperiment hier völlig überein.

Ingerslev (Kopenhagen) stimmt darin mit Halbertsma überein, dass Compression der Uretheren Albuminurie (was auch Runeberg nachwies) und Eklampsie bewirken kann. Aber man muss sich doch wundern, dass bei dem geschilderten anatomischen Verhalten der Uretheren die Compression derselben und Eklampsie nicht öfter vorkommen. Die Dilatation der Uretheren, sowie die Hydronephrose bei Wöchnerinnen ist übrigens schon vor 23 Jahren von Stadfeldt beschrieben worden, und zwar, ohne dass bei den von ihm gesehenen Fällen Albuminurie vorhanden gewesen wäre. Auch in den angezogenen Fällen Löhlein's fehlte nicht weniger als 24 Mal die Dilatation der Uretheren. In der Mehrzahl der Fälle von Eklampsie wissen wir auch gar nicht, ob vorher Albuminurie bestand. In gerslev glaubt allerdings, dass diese oft Folge einer Reflexneurose sein könne. Auch das ist keineswegs durch zahlreiche Untersuchungen bestätigt, dass Albuminurie bei Primiparen und Gemini häufiger vorkommt.

Bar (Paris) hat bei Cornil sich viel mit der betreffenden Frage beschäftigt. Die Pathogenese der Albuminurie ist zu trennen von derjenigen der Eklampsie. Halbertsma lege das Hauptgewicht auf die Compression der Uretheren als Ursache für die Eklampsie, weniger als Ursache für die Albuminurie. Vielleicht sind so die Fälle zu erklären, wo zuerst allgemeine Oedeme bestehen ohne Albuminurie, worauf diese dann zugleich mit Eklampsie auftritt. Es kommt ferner vor, dass mikroskopische Zeichen der Nephritis (Nierenzellen) gefunden werden ohne Albuminurie und umgekehrt. Hat eine Frau in den letzten Monaten der Schwangerschaft Albuminurie, so kann sie Nephritis haben. Daneben kann Albuminurie noch durch die verschiedensten Ursachen bedingt sein, durch Circulationsstörungen, nervöse Einflüisse, Ueberanstrengungen u. s. w. Die Albuminurie ist eben nur Symptom, keine Krankheit. Vorübergehende Albuminurie und solche, welche auf Morbus Brighti beruht, lassen sich freilich oft schwer unterscheiden. Bouchard hat die Befunde des Urins in solchen Fällen näher 
geprüft und gewisse Unterscheidungsmerkmale angegeben. Man muss offenbar mehrere Sorten Albumin unterscheiden, Paralbumin und Metalbumin u. s. w.; so lange aber deren Auseinanderhaltung nicht möglich ist, können wir auch nicht ohne Weiteres annehmen, dass es nur eine Albuminurie gebe.

Halbertsma (zum Schlusswort) ist mit Bar in vielen Punkten einverstanden, besonders in dem, dass die Compression der Uretheren eben nur e in e Ursache der Albuminurie ist, aber sie sei die häufigste. Auf die Frage von Ingerslev, weshalb Oedem und Albuminurie nicht noch häufiger auftrete, erwidere er, dass dies eben erst dann geschehe, wenn die Compression der Uretheren und die secundären Circulationsstörungen einen höheren Grad angenommen hätten. Man scheine sich gegen seine Erklärungsweise deshalb nicht zustimmend zu verhalten, weil sie zu einfach sei, und doch sei das Einfache das Beste. Dass seine Theorie noch weiter geprüft werden müsse, gestehe er gern zu.

10) A. Martin (Berlin): Zur Diagnose und Behandlung der Tubenkrankheiten.

Der modernen Gynäkologie ist schon oft der Vorwurf gemacht worden, dass sie sich zu sehr mit der Technik, mit der Ausbildung der operativen Richtung befasse. Und doch ist es die Ausbildung der Technik der Untersuchung, die Diagnostik, welche dazu hinüberleitete. Ganz besonders kommt diese jetzt und in Zukunft den Erkrankungen der Uterusadnexe zu Gute. Hennig und Bandl bringen über diese vortreffliche anatomische, aber sehr dürftige diagnostische Angaben. In dem Lehrbuch der Gynäkologie von Emmet fehlen die Tubenerkrankungen gänzlich. Die Tubensondirungen hält Martin, wenigstens in der Mehrzahl der Fälle, für falsche Wege. Das von Hausammann (Frankenhäu ser's Klinik) zur Erleichterung der Diagnose bei Tubengeschwülsten empfohlene Ausdrücken nach dem Uterus hin hält Martin für zu gefährlich. Er giebt an, dass selbst die normale Tube sehr wohl zu fühlen und, besonders leicht in Narkose, von den Uterushörnern aus nach den Ovarien hin $z \mathfrak{u}$ verfolgen ist. Unter: 1000 Frauen fand er $63 \mathrm{Mal}$ die Tube nachweislich erkrankt, meist an Salpingitis, kenntlich durch Schwellung, grössere Weichheit, bei höheren Graden durch Bildung gewundener, schlauchartiger vom Uterinende her anschwellender Tumoren. Es sind besonders zwei Arten von Tubenerkrankungen, die er hier hervorheben möchte: 1) die häufige Miterkrankung der Tube bei Ovariencysten, 2) die relative Häufigkeit der Tubo-Ovarialcysten. 
306 Verhandlungen der gynäkolog. Section des VIII. internationalen

Die Therapie anlangend, so kann langwierige palliative Behandlung wohl günstige Erfolge aufweisen, aber es bleiben genug Fälle übrig, wo nichts erreicht wird und die Tubensäcke entfernt werden müssen. Er hat $18 \mathrm{Mal}$ grössere-Tubentumoren operirt, nach Befreiung von Adhäsionen mit Ausschälung, oder, wenn der Sack nicht ganz entfernt werden konnte, mit Vernähung des verkleinerten Sackes und Drainage nach der Scheide. Die Operation bietet schwierigere Verhältnisse dar, als die Ovariotomie. Während ihm von 100 Ovariotomien nur 3 Fälle starben, verlor er von jenen 18 Fällen 5, davon 4 an Sepsis.

Die Tubarschwangerschaft anlangend, so ist Mart in mit J. Veit für frühzeitige Entfernung des Tubarsackes durch die Laparatomie. Rechtzeitige Vornahme der Operation wird allerdings erschwert durch die häufige Symptomlosigkeit des Zustandes. Er erzählt einen Fall, wo auch die Berstung ohne bedrohliche Erscheinungen vor sich gegangen war: bei der Laparatomie fand sich der plattgedrückte Fötus dem Blutsack anliegend.

Kugelmann (Hannover) freut sich über die Fortschritte in der Diagnostik der Tubenkrankheiten und hebt das häufige Zusammenvorkommen solcher mit Parametritis hervor.

Kaltenbach (Giessen) bestätigt die Möglichkeit der Betastung normaler Tuben. Wichtiger sei natürlich die Diagnostik der Tubentumoren. Charakteristisch für dieselben ist besonders die Rosenkranzform mit gleichzeitiger U-förmiger Biegung des aufgetriebenen und oft von Pseudomembranen dicht umhüllten Tubenrohres, ferner unterbrochene Abschnürung einzelner Segmente desselben zu keulenförmigen oder rundlicben Säcken, welche nach dem Abdominalende immer grösser werden. Erzählt einen Fall, wo er eine derartig gestaltete Pyosalpinx für ein kleines Dermoidkystom gehalten hat. Von besonderer Bedeutung sind auch kolikartige, intermittirende Schmerzen, welche auf ein Ausgehen von einem mit muskulärer Wandung begabten Organ, wie die Tube, hinweisen.

Sänger (Leipzig) lenkt die Aufmerksamkeit auf die eminente ätiologische und diagnostische Bedeutung der Gonorrhoe für die schwereren Erkrankungen der Uterusanhänge, besonders der Tuben. Erklärt die vollkommen zutreffenden Anschauungen Nöggerath's über diesen Gegenstand für noch lange nicht bekannt und anerkannt genug unter Aerzten wie Laien. Er fand in zahlreichen Fällen von chronischer Gonorrhoe, eben durch diese darauf hin- 
geleitet, leicht palpable Erkrankungen der Tuben und Ovarien. Er hat $4 \mathrm{Mal}$ wegen solcher die operative Entfernung der Uterusadnexe vorgenommen, $2 \mathrm{Mal}$ wegen Pyosalpinx aus Perioophoritis, $2 \mathrm{Mal}$ wegen, chronischer Salpingitis, Perisalpingitis und Perioophoritis. In 2 Fällen war je ein Ovarium noch in eine apfelgrosse Cyste verwandelt. Heilung in allen Fällen, doch $2 \mathrm{Mal}$ nach Exsudatbildung. S. bezeichnet die Operation "nicht als Castration und nicht als Salpingotomie, sondern als ,Exstirpation der Uterusanhänge", da er, um die Tuben vollständig wegnehmen zu können, stets den grössten Theil der Ligamenta lata, 1 Mal auch beide Uterushörner mit entfernte.

Hegar (Freiburg i/B.) wundert sich, dass Martin nicht auch auf die Untersuchung per rectum aufmerksam gemacht habe. Man fühlt hierbei schon unter normalen Verhältnissen zwei Stränge, der eine, kürzere, erkennbar als Ligamentum ovarii, der andere, längere, gewundene als die Tube. Der für diese charakteristische Rosenkranz, die stellenweisen Anschwellungen bei Tubentumoren sind per rectum ausgezeichnet zu fühlen; ist die Tube mit dem Ligamentum latum, dem Ovarium, entzündlichen Auflagerungen zu einem wirren Convolut zusammengebacken, lässt sich natürlich nichts deutlich unterscheiden. Von pathognostischer Bedeutung für tubäre Erkrankung erachtet er mit Kaltenbach den eigenthümlichen, klopfenden Schmerz in der Inguinalgegend, auf welchen Prochownick zuerst aufmerksam gemacht hat. Dass die Tubenerkrankungen meist von Tripper herrühren, ist ausgemacht, doch entstehen sie oft auch im Anschluss an einen Abortus, an ein Puerperium. Die Heilungsprognose ist für chronische Fälle eine sehr schlechte, in frischen ist aber Heilung möglich. Er erzählt einen Fall von frischer, profuser Gonorrhoe mit Sterilität, wo nach Ausätzung des Uterus mit Salpetersäure Heilung und Conception erfolgte. Dies allerdings der einzige derartige Fall, der ihm vorgekommen sei. In vielen Fällen bleibt nichts übrig, als die operative Behandlung, welche grosse Schwierigkeiten darbiete. Zur Ligatur hat er in neuerer Zeit elastisches Material angewandt. Die Prognose sei aber auch für die Zeit nach der Operation keine günstige, indem sich oft langwierige Peri- und Parametritiden anschlössen.

Simps on (Edinburgh): Auch die bipolare Untersuchung durch Blase und Scheide, Blase und Rectum gestatte, die Tuben zu fühlen. Erschwert werde die Erkennung derselben in Erkrankungsfallen durch Verwachsung mit benachbarten krankhaften 
308 Verhandlungen der gynäkolog. Section des VIII. internationalen

Gebilden (Cysten, Abscessen u. s. w.), sowie durch Lageveränderungen. Empfiehlt für zweifelhafte Fälle die diagnostische Punction von der Scheide aus.

Martin (zum Schlusswort) schliesst sich den Ausführungen von Kaltenbach, Hegar, Sänger vollkommen an, glaubt sich aber doch bezüglich der Prognose günstiger äussern zu müssen. Sah $2 \mathrm{Mal}$ Conception in Fällen wie demjenigen von Hegar. Die von Simpson befürwortete Punction verwirft er.

\section{Sitzung vom 14. August Vormittags.}

Vorsitzender: Breisky (Prag).

11) Engelmann (St. Louis): Ueber Periodicität der Symptome bei Gebärmutterleiden.

Nichts erscheint natürlicher, als dass solche in Malariagegenden wie das Mississippithal, woselbst er prakticirt, ungemein häufig auftritt, wie sie auch in die Symptome anderer Krankheiten unverkennbar eingreift. Die Malaria complicirt sich mit der Grundkrankheit, so auch bei Uterinleiden. Während nun die von der menstrualen Fluxion herrührenden Beschwerden stets $\mathrm{zu}$ der dieser zukommenden Zeit erscheinen, wenn anders sie nicht in der Intermenstrualzeit auftreten, sind die von der Malaria herrührenden Symptome wie Fieber, Schmerzen im Unterleibe, Neuralgien, vermehrter Ausfluss etc. nicht an den menstrualen Typus gebunden, auch nicht anhaltende wie jene, sondern sie kommen besonders vor, aber auch nach und zwischen den Perioden zu bestimmten Stunden des Tages, um nach nur stundenlanger Dauer am nächsten Tage wiederzukehren, bis zum Aufhören des Anfalls. Endometritis, „Endocervicitis"6, „Cellulitis", Carcinom etc. lassen deutlich diese, malarial recurrencers erkennen. Von grosser therapeutischer Wichtigkeit ist dabei, dass kein interperiodisch verabreichtes Mittel wie Chinin, Arsen etc. im Stande ist, diese Symptome abzuschneiden, bevor das Grundleiden gehoben ist. Die Erklärung dürfte in einer Steigerung der ovariellen und uterinen Congestion durch das Malariagift zu suchen sein.

Kugelmann (Hannover) erzählt einen Fall von wehenartigen, periodischen Schmerzen bei Endometritis, combinirt mit Malaria. Heilung durch Behandlung des Catarrhs bei gleichzeitigem Chiningebrauch. 
Gordon (Portland) lebt in ziemlich malariafreier Gegend und hat keine solche hartnäckige Fälle gesehen, benutzt aber die Mittheilung zu einem Hinweis auf Tait's Theorie, wonach Ovulation und Menstruation zeitlich nicht zusammenfielen: vielleicht hingen die periodischen Beschwerden vor dem Eintritt der Menstruation von der Ovulation ab.

Hegar (Freiburg i/B.): Man sieht Periodicität vielfach bei Erkrankungen der Sexualorgane, wobei dieselbe nicht durch letztere bedingt $\mathrm{zu}$ sein braucht. Periodische Schmerzen bei Endometritis, Carcinom hat schon Simpson d. $\ddot{A}$. hervorgehoben, ja er hat sie für das Carcinom sogar als pathognomonisch angesehen. Die Periodicität kann bedingt sein durch Ansammlungen, welche innerhalb gewisser Zeiten zu Entleerungen führen müssen. So ist auch die Defäcation eine periodische Function. Es gilt in jedem Falle der speciellen Ursache nachzuspüren. Wenn z. B. vor der Menstruation gewisse krankhafte Zustände auftreten wie Conjunctivitis, Verschlimmerung einer Mastitis, periodisches Nasenbluten etc., so kann die Ursache davon der erhöhte Blutdruck sein, wie er bei dem Vorgange der Menstruation sich geltend macht. Die Irrenärzte kennen ein periodisches Irresein, welches mit den Menses eintritt: diese geben nur den Anlass zur „Explosion", welche auch während und nach den Menses erfolgen kann, wobei dann der verminderte Blutdruck im Gehirn, relative Anämie desselben, die Ursache abgiebt.

Engelmann (zum Schlusswort) kann Gordon nicht beipflichten. - Betont, dass er durchaus nicht die gewöhnlichen Neurosen während der Menses und in der Intermenstrualzeit, auch nicht die periodischen Schmerzen bei Carcinom gemeint habe. Er sprach nur von solchen durch Malaria bedingten Schmerzen, die in derselben Zeit des Monats erscheinen, also bald vor, bald während, bald nach den Menses, stets zur gleichen Tageszeit und nur für einige Stunden, Schmerzen, welche nicht eher von Chinin beeinflusst werden als bis das Grundleiden gehoben ist. Solche Personen dürfen nicht unaufhörlich mit Chinin behandelt werden, wie es aus Missverständniss der Sache meist geschieht.

12) Stadfeldt (Kopenhagen): Die Behandlung der $\mathrm{Nachgeburtsperiode.}$

Hat sich namentlich die Aufgabe gestellt auszufinden, welches Resultat die drei Hauptmethoden : die exspectative Behandlung, die Credésche Methode und der sogenannte Dubliner Handgriff ergeben haben. 
310 Verhandlungen der gynäkolog. Section des VIII. internationalen

I. Bei der von S. angewandten Form der exspectativen Behandlung wurde die spontane Lösung der Nachgeburt und ihr spontanes Herabsinken in die Scheide abgewartet, doch in der Regel nicht länger als einige Stunden, wenn nicht ernstliche Blutungen frühere Entfernung des Uterusinhaltes nöthig machten. II. Der Credé 'sche Handgriff wurde in vorschriftsmässiger Weise ausgeïbt und die Nachgeburt gewöhnlich mit der 3. Wehe herausgefördert. - III. Der von verschiedenen Autoren etwas abweichend aufgefasste Dubliner Handgriff wurde so vorgenommen, dass auf Reibungen des Fundus wie beim ersten Tempo des Credé'schen Handgriffs das meiste Gewicht gelegt wurde: war die Nachgeburt nach einiger Zeit dann in die Scheide herabgesunken, so wurde sie bei gleichzeitigem leichtem Zug an der Nabelschnur mit zwei Fingern herausgenommen. Nur wenn die Nachgeburt nach Ablauf einer halben Stunde noch nicht herabgesunken war, wurde auch ein auf den Fundus wirkender Druck angewandt. In beifolgender Tabelle sind die erzielten Resultate verzeichnet. Alle Reihen entfallen unter antiseptische Behandlung, welche 1869 im Kopenhagener Gebärhaus eingeführt und seitdem mit steigender Energie angewandt worden ist. Nicht mit verzeichnet sind Fälle von frühzeitigen Aborten, von Placenta praevia, von putridem Uterusinhalt, von einzelnen besonders schwierigen Geburten, wo die Herausnahme der Placenta gleich nach Geburt des Kindes angezeigt war.

Tabelle.

\begin{tabular}{|c|c|c|c|c|}
\hline Behandelte & $\begin{array}{l}\text { I. } \\
\text { Exspectativ. v. } \\
\text { 1.April 1873 bis } \\
\text { 31. März 1877. } \\
\text { 1780 Kreissende. }\end{array}$ & $\begin{array}{c}\text { II. } \\
\text { Credé'sche Me- } \\
\text { thode, v.1. Sept. } \\
1877 \text { bis 1. Feb. } \\
1881 . \\
1611 \text { Kreissende. }\end{array}$ & $\begin{array}{c}\text { III. } \\
\text { DublinerHand- } \\
\text { griff, v. 12. Mai } \\
\text { 1882 bis 15.Feb. } \\
1884 . \\
971 \text { Kreissende. }\end{array}$ & $\begin{array}{c}\text { IV. } \\
\text { Exspectativ, } \\
\text { ivom 16. Feb.bis } \\
\text { 30. Mai } 1884 . \\
\text { 198 Kreissende. }\end{array}$ \\
\hline $\begin{array}{l}\text { Blutungen während der } \\
\text { Ausstossung der } \\
\text { Nachgeburt . . . }\end{array}$ & $104 \mathrm{Mal}=5,8^{\circ} \%$ & $36 \mathrm{MaI}=2,3^{0} \%$ & $13 \mathrm{Mal}=1,3 \%$ & $6 \mathrm{Mal}=3 \%$ \\
\hline $\begin{array}{l}\text { Lösung und Entfernung } \\
\text { der Placenta }\end{array}$ & $24 \mathrm{Mal}=1,3 \%$ & $10 \mathrm{Mal}=0,6 \%$ & $8 \mathrm{Mal}=0,8 \%$ & $2 \mathrm{Mal}=1 \%$ \\
\hline $\begin{array}{l}\text { Abreissen von Eihäuten } \\
\text { oder Placentartheilen }\end{array}$ & $32 \mathrm{Mal}=1,8 \%$ & $37 \mathrm{Mal}=2,3 \%$ & $17 \mathrm{Mal}=1,8 \%$ & $7 \mathrm{Mal}=1,5 \%$ \\
\hline $\begin{array}{l}\text { Blutung während des } \\
\text { Wochenbettes . }\end{array}$ & $14 \mathrm{Mal}=0,8 \%$ & $5 \mathrm{Mal}=0,3 \%$ & $3 \mathrm{Mal}=0,3 \%$ & $0=0 \%$ \\
\hline $\begin{array}{l}\text { Die puerperale Morbi- } \\
\text { dität } \cdot \cdot \cdot \cdot \cdot \cdot\end{array}$ & $438=24^{0} \%$ & $295=18,3 \%$ & $118=12 \%$ & $23=12 \%$ \\
\hline $\begin{array}{l}\text { Die puerperale Morta- } \\
\text { lität } . . .\end{array}$ & $34=1,9 \%$ & $10=0,6 \%$ & $\mathbf{T}=0,7 \%$ & $\mathbf{1}=0, \mathbf{5}^{\%} \%$ \\
\hline
\end{tabular}


Sowohl in Hinblick auf die Resultate der früheren wie die der letzteren, kleineren Versuchsreihe mit dem exspectativen Verfahren stellt sich die Credé'sche Methode günstig. Abreissen und Retention von Eitheilen kommen allerdings etwas häufiger vor, aber gross ist das Uebergewicht nicht und wird diese Gefahr der Expression durch die Antisepsis paralysirt. S. meint, dass die Angreifer der Credé'schen Methode ungerecht gegen dieselbe sind: sie verweilen zu sehr bei den Nachtheilen derselben, heben aber nicht genug hervor, dass sie die Gefahr entfernt, welche die exspectative Methode während der Wartezeit namentlich ausserhalb der Gebärhäuser mit sich führt. Da sie aber bei guter und unschädlicher Anwendung viel Intelligenz und Accuratesse erfordert und in der Hand des weniger Geübten Gefahren mit sich führen kann (z. B. Inversio uteri, welche er $1 \mathrm{Mal}$ von seiner Oberhebamme bewirken sah), so wird sie nicht die Hebammen gelehrt, wohl aber der Dubliner Handgriff, welcher sich leicht erlernen lässt, die Gefahr des Blutsturzes und der Retentio placentae vermindert, der Gefahr des Abreissens und der Verhaltung von Eitheilen vorbeugt. Was schliesslich die Bedeutung der verschiedenen Behandlungsweisen für die puerperale Morbidität und Mortalität anlangt, so glaubt S. nicht, dass die Retention von Eitheilen, namentlich von Decidua heutzutage von besonderem Einfluss auf jene sei; richte man die Prophylaxe auf diese unwesentliche Gefahr, so entferne man sich leicht von der wesentlichsten Prophylaxe: die Bestrebung, die Geburt überhaupt aseptisch zu gestalten. „Trägt man Sorge dafür, dass die Finger, der Geburtsweg etc. während der Geburt aseptisch sind, wendet man kräftige Desinfection an, wo Grund vorhanden ist, die Einführung septischen Stoffes zu fürchten, und enthält man sich übrigens unnöthiger Polypragmasie während des Wochenbettes, so werden selbst grosse Deciduareste im Uterus nicht septisch werden und viel weniger noch Veranlassung zur Selbstinfection geben."

Le opold (Dresden) stellt sich mit Zurückweisung der gegen die Credé'sche Methode gerichteten Angriffe ganz auf die Seite Stadfeldt's. Was die spontane Lösung der Placenta betrifft, wie er sie bei seinen 3 Kaiserschnittfällen direct beobachtete, so zeigen sich an der Placentarstelle keine weiten klaffenden Gefässe, sondern ganz glatte Decidua, in deren Drüsenschicht die Lösung erfolgt. Der retroplacentare Bluterguss ist nicht die Regel. - Ist die Placenta durch den Credé 'schen Handgriff bis in die Scheide geboren, dann darf sie allenfalls durch leisen Zug an der Nabel- 
schnur vollends entfernt werden, wie es der Dubliner Handgriff vorschreibt, Abreissung von Eihäuten kommt wohl vor, ist aber ohne schlimme Bedeutung. Auch die Blutungen bilden nur ein seltenes Vorkommniss bei der Cre dé'schen Methode. Die Hebammen soll die exspectative Methode nicht gelehrt werden; auch der praktische Arzt werde stets rasche Beendigung der Nachgeburtsperiode durch die Expressionsmethode vorziehen.

Simpson (Edinburgh): Die ,deutsche“ Behandlung der dritten Periode hat auch auf die britische Einfluss gewonnen und diese zu einer mehr activen gemacht. Credé's Praxis ist vielfach missverstanden worden. Auch S. hat die exspectative Methode in der Edinburgher Anstalt versucht und hält sie wohl für Kliniken, aber nicht für die Praxis geeignet. Die Beobachtung des physiologischen Vorganges der Lösung und Ausstossung der Nachgeburt, wie sie Ahlfeld und Leopold studirten, ist sehr werthroll. Auch Barbour hat jüngst derartige Untersuchungen an einem. Porro-Uterus angestellt und stimmten dieselben mit denen Leopold's überein.

Stadfeldt (zum Schlusswort) constatirt die Thatsache, dass keine Gegner aufgetreten seien, denen er zu erwidern hätte. -

13) Paul Bar (Paris). Der Basiotribe von Tarnier.

Das 1883 erfundene Instrument soll vor Allem die Basis cranii zertrümmern, daher sein Name. Der Kephalothrypter Ba udelocque's zertrümmert wohl den Kopf, wenn er gut gefasst ist, aber gerade das Fassen und dann das Ausziehen, ohne dass das Instrument ausgleitet, bildet die Schwierigkeit. Der Kranioklast Simpson's in seinen Verbesserungen von Braun, Fabbri etc., der Forceps-scie, die Instrumente von Lazarewitsch (s. unten) geben gute, doch nicht befriedigende Resultate. Einen über dem Beckeneingang stehenden Kopf vermag aber der Kranioklast weder zu verkleinern, noch durchzuziehen, es sei denn, dass es gelingt, die Basis cranii zu erfassen: er ist ein gutes Zug-, aber ein schlechtes Zertrümmerungsinstrument. Der Basiotribe soll den gerügten Mängeln abhelfen. Er besteht aus 3 Branchen, einer centralen und zwei seitlichen. Die centrale, mittlere Branche ist ein Perforator in Bohrerform, welcher bei Hinterhauptsstellung bis zum Stimbein vorgetrieben werden soll. Wird nun die einem schmalen, gefensterten Zangenblatte ähnliche, mit einem Ausschnitt versehene linke Branche durch Einschnappen in eine geknöpfte Achse des Perforatorium geschlossen, so ist das Instrument gleich 
einem Kranioklast zu gebrauchen. ${ }^{1}$ ) Zeigt das dermaassen angelegte Instrument Neigung zum Abgleiten, so kann man das Perforatorium in das Keilbein einbohren. Will man den Kopf noch weiter verkleinern, so wird die rechte, dritte Branche eingeführt. Dieselbe ist etwas länger als die linke und wird mittels Ausschnitt und geknöpfter Achse, die sich auf der zweiten Branche befindet, geschlossen. Jetzt ist das Instrument ein Kephalothrypter, dessen Halt am Kopfe durch die centrale Branche, den Bohrer, gesichert wird. ${ }^{2}$ ) Das Diagramm des zertrümmerten Kopfes ist $\mathbf{8}$ förmig. Bei einem Fall von Beckenenge mit $6 \mathrm{~cm}$ Conjugata vera gelang es binnen 20 Minuten die Basiotripsie zu vollenden (Frau inficirt aufgenommen, starb nach zwei Tagen). Schildert noch weitere Fälle, um die specielle Anwendung in verschiedenen Kindeslagen zu zeigen. Bei Gesichtslagen tritt stets das Kinn in das Fenster einer Aussenbranche, wodurch das Instrument an Halt gewinnt, und empfiehlt B. dasselbe, weil es die Vortheile der Kranioklasie und Kephalothrypsie in sich vereinigt.

Simpson (Edinburgh) erkennt in dem Basiotribe ein vorzügliches Instrument zur Zertrümmerung und Ausziehung des Kopfes, möchte aber doch das Hauptgewicht legen auf die intracranielle Zerbrechung der Basis cranii. Ist diese erreicht, dann bietet der Kranioklast für die Extraction geringere Schwierigkeit und geringere Gefahren, da er von den Weichtheilen des Kopfes gedeckt ist und bei seiner Anwendung Infectionskeime nicht so leicht tiefer in den Uterus geschleppt werden. Den genannten Zweck lässt der von ihm construirte Basilyst vollkommen erreichen, so dass die Extraction sogar nur mit dem Finger besorgt werden kann.

P. Müller (Bern) äussert einige theoretische Bedenken gegen das neue Instrument. Sieht den Vortheil des Kranioklasten darin, dass er den Kopf langzieht, verschmälert und so leicht zu extrahiren gestattet. Der Kephalothrypter jedoch bewirkt eine Formänderung des Kopfes, wobei dieser im geraden Durchmesser des Beckens verlängert wird. Dasselbe muss mit dem Basiotribe der Fall sein, da er, mit drei Blättern angelegt, Kephalothrypter ist.

1) Hat aber mehr Aehnlichkeit mit dem Transforateur von Hubert sen. (s. Wasseige, Opérations obstétricales p. 278).

2) Vgl. die ähnlichen Instrumente von Valette und den Gebrüdern Collini bei Wasseige (l. c. p. 301 und 302). 
314 Verhandlungen der gynäkolog. Section des VIIT. internationalen

In Fällen von $6 \mathrm{~cm}$ Conjugata vera und darunter hält ex den Kaiserschnitt für vorzuziehen.

Lazarewitsch (Charkow): Für Fälle von Hinterhauptsstellung und Stand des Kopfes über dem Kleinbecken, wo die Kranioklasie nicht anwendbar ist, für die Kraniotomie bei höheren Graden der Beckenenge hat L. zwei Instrumente construirt, welche gestatten, förmlich Stïcke des Kopfes abzuschneiden und einzeln zu entfernen. Das eine besteht aus einer kurzen starken Scheere, etwa von Gestalt einer Gypsscheere, welche durch eine Schraubenvorrichtung geöffnet und geschlossen werden kann. Die mittels derselben abgetrennten Theile des Kopfes werden von einem ähnlichen, scheerenartigen Instrumente mit stumpfen Branchen gefasst und ausgezogen.

Jennings (London): Ein gewöhnlicher Forceps kann nach der Perforation hinreichen, den Kopf auszuziehen. Man bedarf gar kein diesen so zermalmendes Instrument wie den Basiotribe. Auch sollte die Zahl derselben nicht weiter vermehrt werden. Empfiehlt besonders die Perforation und nachherige Anlegung des Craniotomieforceps (R. Barnes) bei Beckenenge bis zu $11 / 2 "$. Darunter Porro!

Bar (zum Schlusswort) bemerlat Simpson gegenüber, dass der Basilyst wohl die Basis cranii verkleinere, aber für eine leichte Fixtraction muss auch das Cranium selbst verkleinert werden. Der Kephalothrypter ist nicht gefährlich, weil er den Kopf aussen fasst, sondern weil man das Instrument stark senken, den Kopf mitsammt diesem gegen die vordere Uterus- und Beckenwand drängen muss, nicht selten mit Uterusruptur. Müller's Einwand, dass der Kopf mit dem Basiotribe nicht langzuziehen sei, hält er nicht für begründet. Die Instrumente von Lazarewitsch kommen auf ein Morcellement des Kopfes hinaus, sie machen nicht eine, sondern ein Dutzend Operationen und erheischen viel längere Zeit infolge dessen. --

\section{Sitzung vom 14. August Nachmittags.}

Vorsitzender: Simpson (Ediaburgh).

14) Lazarewitsch (Charkow): Die angeborenen Seitwärtslagerungen der Gebärmutter in ihrer Beziehung zu dem Mechanismus der Geburt und zu den periuterinen Geschwülsten. 
Die angeborenen Seitwärtslagerungen der Gebärmutter sind häufig, namentlich die nach links. Sie sind accidentelle und können in andere Lagerungen übergehen, wenn sie. durch Geschwïlste bedingt sind. Wenn sie aber von Entwickelungsstörungen des breiten Bandes oder der Seitenwand der Scheide abhängen, so sind sie unveränderlich, auch wenn periuterine Geschwülste auftreten, ebenso auch in der Schwangerschaft.

Bei ausgesprochener Seitwärtslagerung wird der Eingang in den Douglas'schen Raum schräg verzogen und das Ovarium der längeren Seite gelangt in diesen, um hier mehr oder minder tief herabzugleiten, während das Ovarium der kürzeren Seite gewöhnlich höher hinaufsteigt. Wenn in dem ersteren Falle das Ovarium cystisch entartet, so verweilt es sehr lange im kleinen Becken und drängt den Uterus nach vorn und oben; in dem zweiten Falle entwickelt sich die Cyste im vornherein im grossen Becken und lässt den Uterus frei. Dem entsprechend findet sich bei Cysten der ersten Art ein langer, bei solchen der zweiten ein kurzer Stiel: weiss man also, welches Ovarium cystisch degenerirt ist, so kann man auf die Länge des Stieles schliessen. - Liegt der Uterus seitwärts, so vermag er sich nicht wohl sei es nach vorn oder rückwärts zu beugen: es muss auch eine Seitwärtsneigung oder -beugung entstehen. Pessarien halten in solchen Fällen schlecht wegen der Ungleichheit der Scheidenwände nach Länge und Wölbung der Scheide. Seitwärtslagerungen bewirken nicht selten fälschlich dem Ovarium zugeschriebene, jedoch vom Uterus ausgehende Schmerzen. - Der Geburtsmechanismus wird sowohl von den Latero-positionen wie Latero-versionen wesentlich beeinflusst, doch in verschiedener Weise. Bei den letzteren behält die Gebärmutter ihre Form und ändert lediglich die Richtung ihrer Achse; bei den ersteren behält der Uterus die Richtung seiner Achse, während sich seine Form ändert, indem er auf der Seite, wo er dem Becken stärker angenähert ist, sich in seinem unteren Segment nur wenig ausdehnen kann, während dieses Segment sich nach der anderen Seite sackartig erweitert. Während der Geburt corrigirt man die Seitwärtsneigung durch entsprechende Lagerung der Frau und durch äussere Handgriffe. Bei Lateropositio uteri genügt es, das Collum mittels Finger nach der Mitte des Beckens zu drängen. -

Leopold Meyer (Kopenhagen) sah bei Sectionen von neugeborenen Mädchen auffallend häufig verschiedene Länge der Ligamenta ovarii, in 58 Fällen nicht weniger als $42 \mathrm{Mal}$. Es muss 


\section{Verhandlungen der gynäkolog. Section des VIII. internationalen}

jedoch wieder ein Ausgleich stattfinden, da eine solche Frequenz bei Erwachsenen nicht zu constatiren ist. Unwahrscheinlich ist, dass die von Lazarewitsch geschilderte einseitige Verkürzung der Scheide die gleiche Ursache habe wie die Verkürzung des Eierstocksbandes.

15) Ingerslev (Kopenhagen): Die Sterblichkeit an Puerperalfieber in den dänischen Provinzialstädten und in Kopenhagen, 1867-1883.

Der Gegenstand des Vortrages ist niedergelegt in einer an die Mitglieder der Section vertheilten Schrift (,LLa mortalité puerpérale des villes de province du Danemark et de Copenhague pendant les années 1867 à 83, présenté au congrès international de médecine de Copenhague en $1884^{\circ}$ ), sowie in zwei früheren Veröffentlichungen, deren Autorreferate sich finden im Centralblatt für Gynäkologie 1880, Nr. 15, S. 355 und 1883, Nr. 51, S. 817.

Bonnaire (Paris) macht statistische Angaben ither die Mortalität der Pariser Maternité seit Einführung der Sublimatbehandlung durch Tarnier (1882). Dank der Verbesserung des antiseptischen Verfahrens (darunter prophylaktische Ausspülung des Uterus post partum mit Sublimat 1:1000), einer zweckmässigeren Bearbeitung der Wäsche durch Ueberhitzen, wurde 1883 bei 1042 Entbindungen eine Mortalität von nur $0,69 \%$ erreicht.

Bar (Paris) hebt die Wichtigkeit hervor, welche die statistischen Arbeiten von Ingerslev gerade für das ,Bureau de l'assistance publique" in Paris als Vorbilder besitzen und demonstrirt im Auftrage von $\mathrm{Budin}$ dessen Intrauterincatheter für Postpartum-Ausspülung. Der Rückfluss der Spülflüssigkeit wird durch eine tiefe Hohlkehle an der unteren Seite des Instrumentes gesichert (s. die Abbildungen S. 31). Material: Metall oder Celluloid. ${ }^{1}$ )

Breisky (Prag): Bonnaire's Angaben geben uns Kenntniss von glänzenden Resultaten des Sublimatverfahrens, aber wie es in Paris gehandhabt werde, erscheine es ihm zu activ. Vor Allem erklärt er sich gegen die prophylaktischen Uterusausspülungen mit Sublimat. Auch ohne solche hat er in seiner Klinik eine Morta-

1) Catheter ähnlicher Construction aus Glas sind auch angegeben von Burgkhardt und Toporski. 
lität nicht über $2 \%$. Im Puerperium lässt er nur bei besonderer Indication intrauterine Injectionen vornehmen.
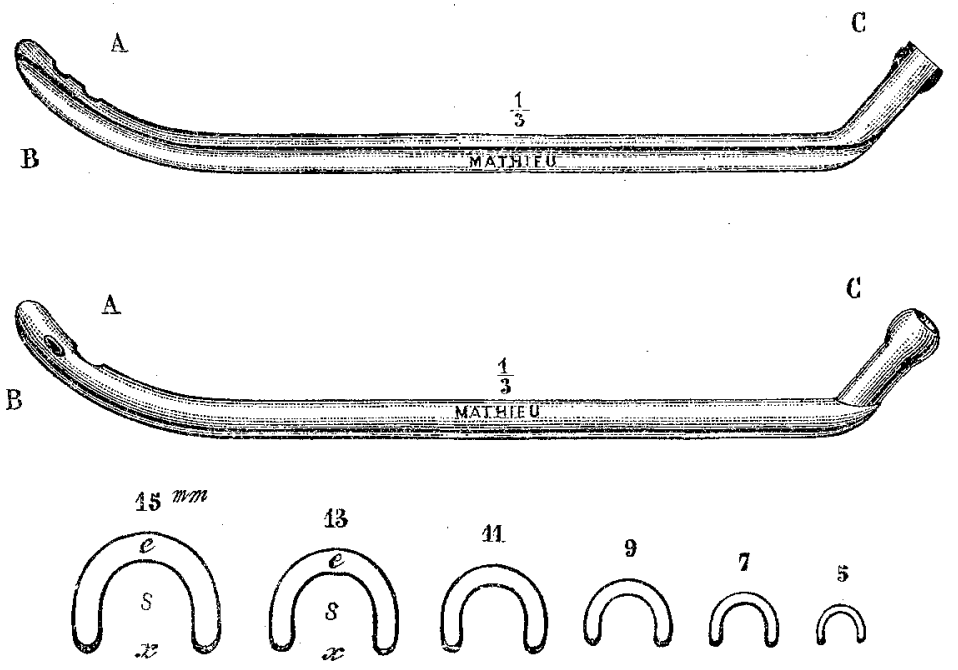

Küstner (Jena) schliesst sich Breisky hinsichtlich der Verwerfung prophylaktischer Uterusausspülungen an; hält die Construction der Budin 'schen Catheter nicht für richtig, gerade weil sie, wie der Bozeman'sche Catheter, einen zu leichten Rückfluss gestatten.

16) Bonnaire (Paris): Demonstration des neuesten Modells der Zange von Tarnier (unter Recapitulation der theoretischen Begründung des Instruments).

Im Wesentlichen gleicht das demonstrirte Modell noch dem vom Referenten in diesem Archive Bd. XVII, S. 407 abgebildeten. Die seitdem vorgenommenen Aenderungen bestehen in Folgendem: Die Zugstiele sind nicht mehr durch Schraubenscharniere an den Löffeln befestigt, sondern sind zum Herausnehmen und leichten Reinigen eingerichtet; sie sind schlanke Stäbe, die sich von innen her durch das Loch am Fensterwinkel des Zangenblattes durchschieben lassen und in einem Knopf endigen, der genau in das Loch eingepasst ist. Die Zugstiele, gegen früher viel schmäler, laufen in einer Rinne längs des Zangenhalses, so dass sie, wenn an diese angedrückt, nicht vorstehen. Das Schloss ist ein excentrisches Achsenschloss (wie an der Zange von Stoltz, Ref.) mit Schraube, welche so eingerichtet ist, dass sie gestattet, den Abstand der Kopfblätter kleiner und grösser zu machen, ohne dass das Schloss geöffnet zu werden braucht. 
Laza rewitsch (Charkow) demonstrirt seinen (schon in London gezeigten) geraden Forceps: die Zange von Tarnier sei im Grunde genommen auch nichts anderes als eine gerade.

Sänger (Leipzig): Das von Tarnier wieder zur Geltung gebrachte Princip der achsengemässen Zangenextraction ist unzweifelhaft richtig. Das Haupthinderniss für eine allgemeine Verbreitung der Zange von Tarnier aber ist die nationale Verschiedenheit der Zangen. Auch das jetzige Modell des Forceps Tarnier werde aus diesem Grunde nicht darauf rechnen können, in Deutschland eingeführt zu werden. Das Wichtigste an dem Instrumente sind die Zugstiele. Es ist das Verdienst Simpson's gezeigt zu haben, dass sich dieselben an jedem Forceps anbringen lassen, ohne dessen Grundform zu ändern, und hat derselbe in der Edinburgher Schulzange combinirt mit modificirten Zugstielen eine vorzügliche englische Achsenzugzange construirt. Redner hat dann für die deutsche Zange ähnliche Nebenapparate angegeben (s. dieses Archiv Bd. XVII, S. 414 und Bd. XX, S. 350), deren Functionirung ihn andauernd aufs höchiste befriedigt.

Simpson (Edinburgh): Seine Bemühungen, den Forceps Tarnier in modificirter Form in England populär zu machen; welche von Sänger hervorgehoben wurden, sind von Erfolg gekrönt worden, indem seine Zange in Edinburgh jetzt allgemein gebraucht wird. Der Praktiker soll nur einen Forceps, den er für alle Fälle verwenden kann, kennen lernen. Hält sein Instrument unbedenklich für dazu völlig geeignet.

17) Cordes (Genf): Ueber Medicamente mit Einwirkung auf den Uterus.

Bespricht unter detaillirter Angabe der Dosirung besonders die Wirkung des Chinins, als eines gleich dem Secale emmenagogischen, sowie des Viburnum prunifolium als eines die Peristaltik des Uterus hemmenden Mittels.

\section{Sitzung vom 15. August Vormittags.}

Vorsitzender: Koeberlé.

18) Hegar (Freiburg i. B.): Castration als Mittel gegen nerröse und psychische Leiden.

Die Folgen der Castration, werde dieselbe unternommen, um ein erkranktes Ovarium zu entfernen oder lediglich zur Anti- 
cipation der Climax, sind stets zweierlè Art, locale für die Genitalien (Aufhören der Menses, Atrophie der Organe u. s. w.) und allgemeine für den übrigen Körper. Da die letzteren weit weniger gekannt sind, so lässt sich auch nicht so leicht eine Indication zur Castration auf dieselben gründen, wie auf die ersteren. Daraus ergiebt sich, dass bei Bestand einer Neurose, deren Zusammenhang mit den Genitalien vermuthet wird, die Castration nur berechtigt ist bei einer nachweisbaren anatomischen Alteration der Ovarien, ferner nur dann, wenn dargethan ist, dass die Neurose von dieser Alteration abhängt. Nun weisen aber bei Neurosen gerade die von den Plexus lumbalis und sacralis ausgehenden Beschwerden (Kreuz-, Leib-, Schenkelschmerzen, Störungen bei der Defäcation, Diurese u. s. w.), die von ihm sogenannten ,Lendenmarksymptome“ auf einen Ursprung von den Genitalien hin. Das Sexualsystem steht mit diesem Abschnitte des Centralnervensystemes und so mit dem gesammten Centralnervensysteme in directem Zusammenhange. So lässt sich auch umgekehrt annehmen, dass daśs Sexualsystem secundär afficirt werden kann durch Erkrankung des Lumbarnervengebietes und des gesammten Nervensystemes. Weitere Stützen für jenen $\mathrm{Zu}$ sammenhang bildet eine bei Neurosen von den Genitalien ausgehende Aura; Einseitigkeit der Neurose bei einseitiger Erkrankung der Sexualorgane; das Auftreten der Neurose während der Menstruation, der Schwangerschaft, überhaupt bei erhöhter Thätigkeit der Sexualorgane; Steigerung der Neurose bei Zunahme der localen Beschwerden von Seiten der Genitalien u. s. w. Am beweisendsten für den Zusammenhang sind die Fälle, wo wir die Entstehung der Neurose und ihre Abhängigkeit von den Sexualorganen direct verfolgen können, z. B. bei Verschwinden der Neurose nach Beseitigung eines Druckes auf den Nervenplexus seitens eines Tumor ovarii, nach Aufrichtung eines retroflectirten eingeklemmten Uterus a. s. w. Ist der Causalnexus nachgewiesen, so ist aber noch nicht gesagt, dass es gelingen werde, die Neurose zu heilen. Am einfachsten liegt die Sache, wenn es sich um einen erkennbaren "Irritationsherd" an den Tuben, den Ovarien handelt, nach dessen Beseitigung die Neurose zu schwinden vermag. Misslicher ist es schon, wenn mehrere Irritationsherde vorhanden sind, z. B. ein stark vergrösserter retroflectirter Uterus. Hier kann man wohl darauf rechnen, dass derselbe sich nach der Castration verkleinere, nicht aber darauf, dass die von der Knickungsstelle ausgehenden Erscheinungen schwinden. Unter 
den bereits gemachten Voraussetzungen ist demnach die Castration bei solchen Neurosen indicirt, welche einer Behandlung durch andersartige mildere Mittel nicht mehr zugänglich sind, welche die Psyche in Mitleidenschaft zogen, welche den Lebensgenuss erheblich stören. (Nähere Ausführungen s. Hegar, die Castration der Frauen; Volkmann's Sammlung klinischer Vorträge $136-138$ u. a. a. 0.)

Die von Hegar erzielten Erfolge sind überraschend günstig, die ungünstig abgelaufenen Fälle aber gerade die lehrreichsten. Misserfolge können aber auch von der Operation selbst herrühren, durch Stumpfexsudate. bedingt sein, welche Fortdauer der Beschwerden, ja sogar Blutung bewirken: die Nervenenden können eben von einem dann dem ovulirenden Organe äquivalenten entzündlichen Knoten weiter gereizt werden. Weiss man doch, dass in der natürlichen. Climax auftretende Geschwülste menstruelle Blutungen wieder hervorrufen können, wie man auch beobachtete, dass mit Perlsucht behaftete Kühe nach der Castration weiter bluteten.

An einem mangelhaften Resultate der Operation kann auch ungenügende Nachbehandlung der Neurose Schuld sein. Dann sind die Beschwerden der Climax untermischt mit Resten der Neurose. Tritt hier noch geeignete Weiterbehandlung ein, so kann das Endresultat doch noch ein günstiges werden. Weitere Ursachen des Misslingens sind Erschlaffung der Bauchdecken, Bauchbrüche: sie bedingen Zerrung der Nerven nicht blos der Bauchwand, sondern auch der Eingeweide, der Nieren, des Mesenterium, der Sexualorgane. Es bilden sich noch wenig näher untersuchte Druckveränderungen im Abdomen aus, welche vorläufig dahin formulirt werden können, dass es zu Herabsetzung des Druckes in der Bauchhöhle und in der Scheide kommt, welche zu hypertrophischen, nicht zu atrophischen Zuständen in genannten Organen führt. Die hier besprochene Frage wird in der Folge noch viel discutirt werden und bewirken, dass wir wieder mehr in Verbindung treten mit den übrigen Fächern der Gesammtmedicin, namentlich der Neuropathologie, welche für die Gynäkologie hierzu die Brücke bildet. Um das Thema aber ganz von der Tagesordnung verschwinden zu lassen, dazu gehörten nicht blos geeignete prophylaktische Maassregeln gegen die Zunahme von neurotischen und Erkrankungen der Sexualorgane, sondern vor Allem eine Aenderung unserer socialen Verhältnisse. 
Koeberlé (Strassburg): Neurosen können nicht nur von den Ovarien, sondern auch vom Uterus, von den äusseren Genitalien herrühren, sowie in socialen Missständen, Fehlern der Erziehung u. s. w. wurzeln. Sie entstehen aus einer Vielheit von Ursachen. Man muss auch umgekehrt fragen, wie viele und was für Erkrankungen der Sexualorgane, besonders der Ovarien, Neurosen zur Folge hatten. Interpellirt deshalb Spencer Wells, $\mathrm{ob}$ er ż. B. bei Ovarientumoren so häufig solche gefunden habe.

Sir Spencer Wells (London) antwortet mit Nein. Auch könne er den Neurosen, welche vom Ovarium ausgehen sollen, keinen ihnen eigenthümlichen Charakter zuerkennen. Hatte vier Mal Gelegenheit, bei Geisteskranken die Ovariotomie zu machen: bei zweien besserte sich die Psychose. Doch sah er auch in einigen Fällen Verschlimmerung der letzteren nach der Operation. Bezweifelt die Häufigkeit des Zusammenhanges von Neurosen mit den Eierstöcken. Weit mehr seien erstere abhängig von Erziehungsfehlern und hygienischen Missgriffen. Keinesfalls sei die, namentlich in Amerika, zu oft geübte Castration bei ganz gesunden Ovarien gerechtfertigt, ebensowenig als z. B. die Castration geisteskranker Männer.

Martineau (Paris) hat als Leiter der grössten gynäkologischen Abtheilung im Hospital Bicêtre sich schon seit langem mit Neurosen der betreffenden Art beschäftigt. Er fand bei schweren Neurosen und hysterischen Zuständen häufig keinerlei Erkrankung der Ovarien, wohl aber solche des Uterus, namentlich chronișche Metritis. Er hält eine ,nervöse Disposition“ für das Primäre; komme dazu eine Erkrankung der Sexualorgane, dann trete ein Ausbruch oder eine Verschlimmeruing der nervösen Symptome ein, welche auf Behandlung des Uterus zurückgingen.

Olshausen (Halle): Die Frage ist unstreitig eine sehr schwierige. Selbst wenn die Gegend der Ovarien hyperästhetisch ist, braucht das nervöse Grundleiden nicht davon auszugehen. Wenn ferner Hegar verlangt, anatomische Alterationen der Eierstöcke palpiren zu können, so wird dies nicht immer möglich sein, wo dieselben doch krank sind. Jedenfalls ist die Indication zur Castration bei nervösen und psychischen Leiden sehr zu beschränken. Seit $21 / 2$ Jahren hat er keine solche Operation mehr gehabt. Ex erzählt einen Fall, wo palpable Aenderungen an den Ovarien nicht nachzuweisen waren und er von den Angehörigen gedrängt wurde, die Castration auszuführen. Der Erfolg war gleich Null, 
ebenso in zwei anderen Fällen, wovon der eine mit periodischer Manie. Diesen gegenüber steht allerdings auch ein Fall mit $\mathrm{Hy}$ stero-Epilepsie, wo nach Entfernung der normalen Ovarien die Anfälle ausblieben, und zwar seit nunmehr $21 / 2$ Jahren. Er hat Grund anzunehmen, in diesem Falle die Ursache der Erkiankung in nervösen und psychischen Einflüssen schon von der Kindheit her, welche die Ovarien erst später in Mitleidenschaft zogen, zu suchen. Man müsse individualisiren und jeden einzelnen Fall für sich abwägen, ehe man zur Operation schreite.

Gusserow (Berlin) theilt Spencer Wells' und Olshau sen's Standpunkt. Man muss auch fragen, wie lange die Heilung vorgehalten habe. Unmittelbar nach einem operativen Eingriffe pflegen die Beschwerden bei Nervösen oft zu schwinden, um später wiederzukommen. Daher muss auch danach geforscht werden, wie es mit Recidiven stehe.

Kugelmann (Hannover) schliesst sich Spencer Wells und Martineau an, rügt den Missbrauch des Ausdruckes „Hysterie", namentlich als Indication zur Castration.

Gordon (Portland): Es braucht und kann nicht immer nachgewiesen werden, dass die Ovarien erkrankt sind, um eine Berechtigung für die Operation zu haben. Hat im vergangenen Jahre sechs Mal operirt, wo dies nicht möglich war, in Fällen von Dysmenorrhoe mit schwerer allgemeiner Nervosität. Die entfernten Ovarien zeigten sich sämmtlich erkrankt, namentlich durch cysto-folliculare Entartung; auch fanden sich mehrmals die Tuben verschlossen. Die Schmerzen and hysterischen. Krisen wurden prompt beseitigt.

Priestley (London) hat in seiner Consultationspraxis viele Fälle schwerer Neurosen gesehen, aber niemals die Castration für indicirt befunden. Da das Nervenleiden häufig centralen Ursprungs sei und die Beschwerden seitens der Ovarien nur Theilerscheinungen ausmachten, so könne die Castration auch keine Heilung des Grundleidens erzielen. Er sah einen Fall, wo nach der Operation die Schmerzen und nervösen Krisen sich verschlimmerten und schliesslich Geisteskrankheit auftrat. Medicamentöse und psychische Behandlung leisteten doch mehr als ihr nachgesagt werde. Besonders rühmt er das Verfahren von Weir Mitchell (eine combinirte diätetische, psychische, elektrische und Massagebehandlung. Ref.) gegen die in Frage kommenden Leiden.

Engelmann (St. Louis): Für Beseitigung einer Neurose kann unter Umständen die Cervixdilatation und Entfernung eines 
Uteruspolypen denselben Effect haben, wie eine Castration. Die Schwierigkeit der Entscheidung für diese liegt in der Diagnose und in der Wahl des richtigen Zeitpunktes. Er stellte für die Vornahme der Operation stets die strengsten Anforderungen, und operirte nur Fälle mit ganz schweren nervösen Beschwerden, die progressiv schlimmer wurden. Die Schwere der Frscheinungen steht dann nicht immer in Verhältniss mit dem Befunde an den Ovarien. Schon die nicht geringe Zahl Fälle von Verschlimmerung der Neurosen nach der Operation weise uns darauf hin, dieses Grenzgebiet der Gynäkologie und Gesammtmedicin noch weiter anzubauen.

Ko eberlé (Strassburg) erwähnt nur, dass er ein Mal eine schwere Hysterica mit Myom operirt habe. (Erfolg?)

Hegar (zum Schlusswort) meint, dass man den Vertretern der Castration doch $\mathrm{zu}$ viel Schlimmes vorwirft. Geringfügige Sachen, wie eine Narbe, eine leichte mechanische Störung u. s. w., können Anlass einer schweren Neurose sein, während diese umgekehrt bei einer grossen Geschwulst fehlen könne. Damit sei nichts gegen die Erspriesslichkeit der Operation bewiesen. War frappirt, dass Olshausen die Indication weiter ausdehnte, als er selbst, da er blos wegen einer allgemeinen Neurose ohne Bestand einer localen Erkrankung operirt habe. Die sichere Basis einer pathologischen Veränderung der Ovarien darf nicht aufgegeben werden. Es verstehe sich von selbst, dass Neurosen auch noch durch andere Behandlungsmethoden, z. B. solche, die gegen den Uterus gerichtet seien, geheilt werden könnten. Es können auch combinirte Ursachen für die Neurose vorhanden sein: dann muss der Factor ausgewählt werden, ohne dessen Beseitigung Heilung nicht möglich ist, und da giebt es Fälle, wo nichts anderes übrig bleibt, als die Ovarien wegrunehmen. Schliesslich erzählt er den einzigen ihm vorgekommenen Fall, wo er wegen einer Psychose operirte: es handelte sich um eine junge Frau mit Vaginismus, welche an einen sterilen Mann verheirathet war. Es bestanden hysterisches Irresein und hühnereigrosse Ovarien. Nach deren Wegnahme trat völlige Heilung ein.

19) Martineau (Paris): Die Bedeutung der Adenolymphangitis uterina für die Entwickelung der sogenannten periuterinen symptome und für die Behandlung der Metritis.

Das Lymphsystem des Uterus und seiner Umgebung spielt 
eine grosse Rolle bei deren entzündlichen Erkrankungen. Die Entzündung des Uterus, sei sie acut oder chronisch, kann nicht bestehen ohne Betheiligung des uterinen und periuterinen Lymphsystems. Die "Adeno-lymphangitis periuterina" ist der Ursprung der als Phlegmone des breiten Bandes, als Phlegmone periuterina, als Perimetritis, als Pelviperitonitis bezeichneten Entzündungen. Daher ist die Bezeichnung derselben als "Adeno-phlegmone“ des breiten Bandes, als „Adeno-phlegmone periuterina“, „Adenopelviperitonitis" eine passendere, da sie an den Ursprung erinnert. Da die ,Adeno-lymphangitis periuterina" gleichen Schritt hält mit der Entzündung des Uterus, so muss jeder Anlass, welcher diese anfacht, jene steigern. So auch ärztliche Eingriffe. Er selbst sah einen Todesfall an eitriger Pelviperitonitis nach Aetzung am Muttermunde. Bei der Behandlung sind neben der Erkrankung des Uterus auch bestehende Constitutionskrankheiten (Chlorose, Syphilis, Tuberkulose) sehr zu berücksichtigen. Unter keinen Umständen darf am Uterus operirt werden, wenn acute „Adeno-lymphangitis" besteht. Erst wenn diese beseitigt wurde, ist energische Behandlung des Uterus durch Scarificationen, Aetzungen, Injectionen am Platze.

20) Apostoli (Paris): Ueber eine neue elektrische Behandlung der Perimetritis durch doppelte Faradisation der Gebärmutter.

Die Technik des Verfahrens besteht darin, dass beide Elektroden in den Uterus eingebracht werden. Starke Stromstärke, kurze Schläge und lange Dauer der Sitzung. Anwendung, selbst bei acuter Perimetritis, stets von Erleichterung gefolgt; rasche Resorption. Wirkung ähnlich wie bei Massage. ${ }^{1}$ )

21) Rothe (Altenburg): Demonstration seines Sims-Speculum zum Selbsthalten.

22) Knowsley Thornton (London): Die frühzeitige Ovariotomie.

Ehe der Frage über die geeignetste Zeit für Vornahme der Ovariotomie nahe getreten werden kann, muss die Thunlichkeit der Punction oder Aspiration entschieden werden.

Spencer Wells, Keith, Thomas und andere hervorragende Ovariotomisten befürworten die Punction; Gross, Emmet, Tait sind dagegen.

1) S. Apostoli, A new method of uterine faradization. Americ. journ. of obstetr. etc., Sept. 1884. 
Die für die Punction in Anspruch genommenen Vortheile erscheinen doch sehr zweifelhaft, wenn man ihre Gefahren, ihre unmittelbare und entferntere Sterblichkeit dagegen hält. Weit günstiger und besser stellt sich die Probeincision. Die Punction sollte nicht nur als unklug, sondern mit Stilling geradezu als „ein Verbrechen“ angesehen werden. Nur ganz wenige Fälle giebt es, wo sie angezeigt sein kann, z. B. wenn man Zeit gewinnen und eine Kranke sich erst erholen lassen will, wenn aus irgend welchen Gründen die Operation nicht ausgeführt werden kann. Die gerade bei Parovarial-Cysten viel empfohlene Punction oder Aspiration mit feinem Troicart habe wohl öfters zur Heilung geführt, sei aber deshalb gefährlich, weil diese Cysten so oft Papillome sind, deren Inhalt dann in die Bauchhöhle verschleppt und dem Bauchfell implantirt würde.

Auch als diagnostisches Hülfsmittel ist die Punction überschätzt worden. Um den von dieser, sowie von dem Weiterwachsthum der Cyste drohenden Gefahren zu entgehen, ist es rathsam, wo es nur angeht, die frühzeitige Ovariotomie vorzunehmen. Der richtige, für dieselbe geeignete Zeitpunkt scheint ihm dann gekommen, wenn die Cyste aus der Beckenhöhle emporgestiegen ist, der vorderen Bauchwand anliegt und diese etwas ausdehnt, so dass die Incision auf die Cystenwand fallen kann und nicht auf die Darmschlingen. Noch frühzeitiger soll nur unter besonderen Umständen, z. B. wegen Blutungen, erheblichen Beschwerden, Einklemmungen u. s. w., operirt werden. Die durch Verzögerung der Operation entstehenden Gefahren sind: Infection des Bauchfelles durch Berstung der Cyste und Austritt von Cysteninhalt, bösartige Degeneration der Cysten, ausgedehnte Adhäsionenbildung, Stieltorsion u. s. w. Unter einer Zahl von über 400 Ovariotomieen des Vortragenden befanden sich nicht weniger als 40 Fälle mit freier Cystenflüssigkeit in der Bauchhöhle und 34 Fälle von Stieltorsion. Die Resultate der Ovariotomie in diesen Fällen waren ganz bemerkenswerth schlechtere. In einer ganzen Anzahl von Fällen mit Cysteninhalt in der Bauchhöhle, welcher oft erst durch Punction hineingelangt war, gingen die Operirten später an Krebs des Bauchfelles und anderen malignen Geschwülsten zu Grunde. Bedenkt man noch die bei ungehemmtem Weiterwachsthum der Cyste dem Allgemeinbefinden drohenden Störungen, die immer lästiger werdenden Beschwerden, die sich mehr und mehr steigernde Schwierigkeit der Operation, so wird man sich gewiss für die frühzeitige Ovariotomie entscheiden, denn' Niemand kann, 
der Zukunft ein günstigerer Augenblick für die Operation darbieten werde. Als die Mortalität derselben noch 20 bis 25 Proc. betrug, da konnte man die frühzeitige Ovariotomie nicht empfehlen, jetzt aber wird sie im Verein mit Unterlassung der Punction sicher dazu beitragen, das Heilungspercent auf ein Maximum von 95 Proc. zu steigem.

Howitz (Kopenhagen) hat nur wenige Einwendungen zu machen. Er möchte der Punction doch einen etwas weiteren Spielraum einräumen. Dieselbe muss unterschieden werden als vorbereitende und als einzige Operation. Als erstere kann sie von Nutzen sein einige Zeit vor der Ovariotomie, um Ascites, Oedeme verschwinden zu lassen; bei acuten Erkrankungen. Als einziger operativer Eingriff kann sie bei sehr grossen, voraussichtlich sehr schwierig zu operirenden Cysten, besonders solchen des breiten Bandes, vorgenommen werden. Es sind genug Fälle bekannt, wo in langen Zwischenräumen wiederholte Punctionen das Wohlbefinden erhielten. Allerdings ist dank der Antisepsis die Mortalität der Ovariotomie auf 8 bis 4 Proc. gesunken: Gerade dieser Umstand. könnte aber Manchen verleiten, die Operation allzufrüh auszuführen, denn es giebt viele Fälle von kleinen, lange Zeit stationär bleibenden Cysten, die oft gar keine Erscheinungen machen. So fand sein Assistent, Dr. Kaarsberg, bei 100 von ihm untersuchten Scortis 17 Mal kleinere Ovariencysten. Dass es aber gerade bei solchen Personen selten zu Ovariotomieen kommt, ist bekannt.

Sir Spencer-Wells (London): Die Mortalität bei Punction kann kaum grösser sein, als die bei natürlichen Geburten, jedenfalls ist sie bedeutend niedriger als 5 Proc., die Minimal-Mortalität der Ovariotomie. Bei wirklieh uniloculären Cysten, besonders solchen des Ligamentum latum, kann die Punction völlig ausreichen. Auch die der Ovariotomie vorausgeschickte Punction ist unbedenklich. Wird, wie nicht so selten, die Operation verweigert, bleibt nichts übrig als die Punction.

Koeberlé (Strassburg): Ist die Cyste grösser, liegt sie der Bauchwand breit an, so ist die Ovariotomie viel leichter, ihre Chancen sind grösser als bei kleinen Cysten, wo man mit den Därmen zu kämpfen hat und auch eine längere Incision machen muss. Fand sehr oft bei Autopsie alter Frauen Cysten, die gar keine Symptmoe gemacht hatten.

Thornton (zum. Schlusswort) freut sich, dass seine Anschauungen, besonders über die frühzeitige Ovariotomie, von denen 
wie Spencer-Wells einmal äusserte, versprechen, dass sich in Howitz's, Wells', Koeberlé's nicht sehr abweichen. Er gebe wohl $\mathrm{zu}$, dass für einzelne dringliche Fälle die Punction zulässig sei, doch während die Patientin bei der Ovariotomie die Aussicht hat, ihr Leiden gänzlich loszuwerden, wird sie bei Punction und abwartender Behandlung damit belassen und Gefahren ausgesetzt, die oft erst später auftreten, nicht unmittelbar nach der Punction.

23) Sänger (Leipzig): Ueber Resection des Peritoneum parietale.

Gegenstand des Vortrages ist die in diesem Archiv, Bd. XXIV, Heft 1 erschienene Abhandlung. ${ }^{1}$ ) $\mathrm{S}$. bemerkt noch, dass ihm auch die von Koeberlé als für die Operation contraindicirten Fälle von gefässreicher Verwachsung eines Myom mit der vorderen Bauchwand als sehr geeignet erschienen für die Resection statt der mühsamen und blutigen Ablösung. Ferner hebt er in Hinweis auf den Vortrag von Miculicz hervor, dass trotz so ausgedehnter Resection des Peritoneum als er sie vorgenommen, es doch nicht nöthig sein dürfte, zu drainiren, da, vollständige Stillung der Blutung vorausgesetzt, wegen rascher Verklebung der Eingeweide mit dem ihnen zugekehrten wie angefrischten Hautlappen es nicht zur Ausbildung eines, ,todten Raumes" kommen kann.

Koeberlé (Strassburg) würde nicht wagen, bei Myomotomie mit parietaler Adhäsion das Bauchfell zu reseciren. Bei den so seltenen Fibromen der Bauchwand hat er eine gewisse Scheu vor der Operation. Er machte dieselbe drei Mal; nur ein Fall, wobei das Bauchfell nicht eröffnet wurde, genas. Diese Art Geschwuilste ist sehr schwer, und nur mit sehr bedeutender Blatung aus ihrer Umgebung, den Muskeln namentlich, auszuschälen, weshalb er die Operation für eine sehr gefährliche ansieht.

Sän ger kann diese Gefährlichkeit bei Anwendung entsprechender Technik nicht anerkennen. Man soll die Geschwulst nicht aus den Muskeln herausschälen, sondern dieselben, da sie ohnedies gewöhnlich verdünnt sind und zurückgelassen keine normale Function mehr entfalten, mit dem Tumor entfernen. Durch partienweise Umstechung der Geschwulstumgebung lässt sich die Exstirpation fast blutlos ausführen, und da sich ergeben hat, dass auch das Peritoneum parietale ohne Gefahr in grösserer Ausdehnung mit weggenommen werden kann, so ist die vollständige Entfernung sehr

1) Die Anmerkung anf S. 4 derselben nehme ich als falsch hiermit zurück. 
umfänglicher Desmoide der Bauchwand gesichert und nicht so schwierig.

24) Gordon (Portland): Die operative Behandlung der Hyperplasie des Uterus und der Scheide, mit besonderer Rücksicht auf die Heilung von Lageveränderungen. Lageveränderungen des Uterus, besonders Retroflexio, stehen in innigem Connex mit chronischer Hyperplasie des Uterus und der Scheide: Heilung der einen ist ohne Behandlung der anderen kaum möglich. An eine Heilung von Retroflexio kann auch so lange nicht gedacht werden, als Einrisse der Cervix, des Perineum nicht wieder ausgeglichen sind. Hat man vor diesen plastischen O'perationen den Uterus in normale Lage gebracht, so kann er, wenn die Patientin längere Zeit gelegen hat, ganz abschwellen und normal gelagert bleiben, auch ohne ein Pessar. Nach plastischer Wiedervereinigung von Scheide und Damm ist auch ein Wall gebildet, auf welchen sich der Uterus stützen kann und der auch einem nothwendig werdenden Pessar Halt gewährt. Ganz besonders wird die Abschwellung des Uterus gefördert durch seitliche Ausschneidung keilförmiger Stücke aus dem Collum uteri und nachfolgende Naht.

Meinert (Dresden) bediente sich in mehreren Fällen von Retroflexio, welche der gewöhnlichen Pessarbehandlung trotzten, eines eigenthümlichen Verfahrens, indem er mittels Silberdraht eine Hartgummiplatte an die hintere Muttermundslippe annähte, auf welche sich dann das Pessar besser stïtzen und den Uterus erheben konnte. Auch hat er solche angenähte Platten mit Intrauterinstiften verbunden tragen lassen.

Engelmann (St. Louis) hat ebenfalls durch Excision von Stücken des Collum, durch plastische Beseitigung vorhandener Dammrisse, durch Normallagerung des entlasteten Uterus so vorzügliche Erfolge erzielt, dass sich Uterus und Scheide fast wie virginal verhielten. Bei Cervixrissen soll stets die Emmet'sche Operation gemacht werden. Unmittelbar nach der Operation, und so lange die Patientin nicht thätig ist, genügt Tamponade des hinteren Scheidengewölbes, den Uterus in der richtigen Lage zu erhalten.

Gordon: Besonders wichtig ist es, wenn, wie bei Dammrissen, die Scheide klafft, ihre Wünde aneinander zu bringen und sie fester zu machen, damit einestheils der Uterus sich auf sie stützen, anderentheils ein Pessar Halt finden könne. 
Sitzung vom 16. Angust 1884 Vormittags.

Vorsitzender: Veniselos (Athen).

25) Werth (Kiel): Die operative Behandlung der Extrauterinschwange rschaft.

I. Auch in den ersten Monaten einer Extrauterinschwangerschaft kommt neben dem Versuche, durch Tödtung der Frucht die Schwangerschaft zum Stillstand und den Fruchtsack zur Verödung zu bringen, die operative Entfernung des letzteren in Frage, wenn Tuben- und Ovarialschwangerschaft vorliegt und eine der Operation günstige Verbindungsweise des Fruchthalters mit den angrenzenden Theilen nachweisbar ist. Die Rücksicht auf die Frucht muss hier stets der für die Mütter nachstehen. Die frühzeitige Operation ist daher gerechtfertigt, weil doch nur in den allerseltensten Fällen ein lebendes Kind durch spätere Gastrotomie herausgefördert wird. Die Frühoperation ist auch indicirt, wenn ein im Douglas'schen Raume liegender Fruchtsack schwere Incarcerationserscheinungen nach sich zieht. (In einem derartigen Falle sah Werth Tod an Perforation der Blase.) Dagegen erscheint die Laparatomie im Anschlusse an erfolgte Ruptur des Fruchtsackes aussichtslos und nach den bisher vorliegenden Erfahrungen ein exspectatives Verhalten den Vorzug zu verdienen.

II. Die Operation bei weiter vorgeschrittener Schwangerschaft und lebender Frucht ergiebt, abgesehen von den seltenen Fällen, wo die anatomischen Verhältnisse eine Entfernung des unverletzten Eies mit sammt dem Fruchtsacke gestatten, bei geringer Aussicht auf Erhaltung des Kindes eine lethale Prognose für die Mutter. Nach Litzmann starben von zehn Fällen von Gastrotomie bei lebender Frucht neun Mütter; von sieben weiteren Fällen, die Werth sammelte, wurde nur eine Mutter erhalten (Fall von Martin). Summa: 17 Fälle mit zwei Heilungen. Es ist deshalb principiell jeder operative Eingriff zu Lebzeiten des Kindes, unternommen in der vorwiegenden Absicht, dieses zu erhalten, zu verwerfen und der Fruchttod abzuwarten. Die dem Fruchttode folgenden Expulsivbewegungen lassen sich in der Regel durch geeignete Maassregeln in Schranken halten.

III. Da die extrauterine Lagerung einer über die ersten Entwickelungsstadien hinausgelangten, abgestorbenen Frucht eine dauernde Gefährdung des mütterlichen Lebens bedingt, so ist unter allen Umständen die operative Entfernung der todten Frucht 
nothwendig. Die Prognose des Eingriffes ist unvergleichlich besser als die der Operation bei lebender Frucht, die richtige Wahl des Zeitpunktes für die Operation vorausgesetzt. Dieselbe soll, wenn irgend möglich, nicht früher stattfinden, bevor auf Verödung des mütterlichen Placentarkreislaufes zu rechnen ist, das ist nicht vor der zehnten bis zwölften Woche nach dem Tode der Frucht.

Litzmann sammelte 23 Fälle von Gastrotomie zwischen der sechsten Woche und einem Jahre nach dem Tode der Frucht: es starben $5=21$ Proc. 25 weitere von Werth zusammengestellte Fälle ergaben acht Todesfälle, wovon vier aus nicht von der Operation abhängigen Ursachen. Die innerhalb der ersten sechs Wochen fallenden Operationen können als ,,intermediäre" bezeichnet werden. Von vier Fällen dieser Art starben drei.

IV. Von grösster Bedeutung für die Wahl des Operationsverfahrens ist die richtige anatomische Grundlage. Primäre Abdominalschwangerschaft ist jedenfalls nicht so häufig als angenommen wird. Die Tubarschwangerschaft kommt auch für die späteren Monate viel öfter vor. Unter 36 Fällen von Laparatomie bei Extrauterinschwangerschaft seit 1880 befanden sich 18, wo sicher keine Abdominalschwangerschaft, und 13, wo Tubenschwangerschaft, drei mit ausgetragener Frucht, bestand. W e rith hat dann noch zwei ältere Fälle aus der Kieler Sammlung als ausgetragene Tubarschwangerschaften nachweisen können, welche bisher für Abdominal- resp. Ovarialschwangerschaften gehalten wurden. Für die Operation selbst kommen zwei Hauptformen der Extrauterinschwangerschaft in Betracht:

1) gestielte, 2) intraligamentöse.

1) Hier handelt es sich um Schwangerschaft im äusseren $\mathrm{Ab}$ schnitte der Tube, um Ovarial- und Tuboovarialschwangerschaft. Fruchtsack hoch, beweglich, scharf contourirt. Becken meist frei. Wenn der Douglas aber eingenommen ist, gewöhnlich Incarcerationserscheinungen. Das Operationsverfahren gleicht hier dem der Ovariotomie, ebenso die Prognose. Bisherige Operationen von Litzmann, Billroth, Knowsley Thornton, Schröder (zwei Fälle).

Auch in diesen Fällen ist die Einnähung des Fruchtsackes sicherer für den Exfolg als die Exstirpation.

2) Wahrscheinlich sämmtlich tubären Ursprunges. Diagnose schwierig. Verhältnisse ähnlich wie bei intraligamentären Ovarialcysten. Radicale Operation hier unmöglich. Als Regel ist 
hier, am besten unter Anwendung des zweizeitigen Verfahrens, die Einnähung des Fruchtsackes in die Bauchwunde festzuhalten. Placenta und Eihäute sind $\mathrm{zu}$ entfernen, wenn Zweifel an der bereits erfolgten Thrombosirung der Placentarsinus nicht besteht. Bei Anwendung streng antiseptischer Cautelen während der Operation und Anlegung eines Occlusivverbandes ist, vorausgesetzt, dass nicht bereits Zersetzung des Fruchtsackinhaltes bestand, weder eine präventive Drainage nach der Vagina hin, noch die Anwendung permanenter Durchrieselung des Sackes erforderlich. Beides ist unbedingt zu unterlassen, ebenso die Ausspülung und prophylaktische Tamponade des Fruchtsackes, wenn bei lebender oder vor kurzem abgestorbener Frucht operirt wurde und künstliche oder spontane Abtrennung der Placenta mit der Gefahr tödtlicher Blutung droht. Dagegen empfiehlt sich unter diesen Umständen die theilweise Ausfüllung des Fruchtsackes mit einer Substanz (Tannin und Acidum salicylicum zu gleichen Theilen, nach Freund), welche eine antiseptische Conservirung der Placenta bis- zu ihrer späteren Ausstossung ermöglicht.

Die Elytrotomie wird nur in seltenen, besonders gearteten Fällen der Gastrotomie vorzuziehen sein.

Ueber die allgemeinere Verwendbarkeit und eventuellen Vorzüge der von Martin empfohlenen partiellen Resection des Fruchtsackes mit Vernähung des Restes und Drainage nach der Vagina hin sind erst weitere Erfahrungen abzuwarten. Eventuell geht es an, die Placenta zurïckzulassen und die Bauchhöhle zu schliessen.

Koeberlé (Strassburg) hat fünf Mal operirt wegen Extrauterinschwangerschaft: in drei von diesen Fällen schwankte aber die Diagnose zwischen Uterus bicornis und Tubarschwangerschaft. Vier Heilungen, ein Todesfall. Gerade bei diesem wurde früh operirt, sechs Wochen nach dem Tode der Frucht. In den anderen Fällen wurde später eingegriffen bis zu 21 Wochen danach. Eine Frau gebar nach der Operation noch zwei Mal. - Der Fötus stirbt an Blutungen zwischen den Eihäuten unter den Expulsivbestrebungen. - Bei der Incision des Sackes riskirt man stets die Placenta zu treffen. Ist daher ebenfalls für möglichst späte Operation und für Zurücklassung der Placenta.

P. Müller (Bern) stimmt Litzmann-Werth bei. Berichtet über drei Fälle.

1) Frischer Fall. Bei der Incision die Placenta getroffen. Tod an Verblutung. 
332 Verhandlangen der gynälkolog. Section des VIII. internationalen

2) Gastrotomie nach längerer Zeit. Heilung.

3) Elytrotomie nach drei Jahren. Heilung.

Macht nur ein en Einwand, weshalb bei lebendem Kinde nicht operirt werden soll. Treten dann keine Rupturen des Fruchtsackes ein? Sind dadurch nicht Gefahren bedingt?

Olshausen (Halle) ist zufällig in der Lage, diese Fragen aufzunehmen. Beobachtete die Frau eines Arztes, welche 14 Tage nach dem Tode eines grossen extrauterin gelegenen Kindes an Ruptur des Fruchtsackes starb.

Sänger (Leipzig): Eine Form der Schwangerschaft ,am unrechten Orte" ist von Werth nicht erwähnt, von Koebexlé nur gestreift worden, welche zwar anatomisch keine extrauterine ist, ihr aber klinisch sehr nahe steht; die Schwangerschaft im rudimentären Nebenhorne eines Uterus bicornis. Sie steht in der Mitte zwischen interstitieller und Tubarschwangerschaft, und könnte als ,gynatretische Schwangerschaft" bezeichnet werden. Sänger hat die bisher beobachteten Fälle gesammelt:

22 Fälle, die durch Ruptur in den ersten sechs Monaten tödtlich endeten.

3 Fälle von Lithopädionbildung, worunter einer bei ausgetragener Frucht.

Zur Operation kamen: 1) Der Fall von Koeberlé (1865), Gastrotomie resp. Kaiserschnitt 21 Monate nach dem Tode der Frucht. Verlöthung des Nebenhornes mit der vorderen Bauchwand. Heilung.

2) 1880. Fall von Salin. Diagnose unter der Operation. Abtragung des Nebenhornes nach Porro (richtiger: „Semi-amputatio uteri bicornis gravidi"). Heilung. Die Frau gebar wieder.

3) 1881. Litzmann-Werth. Frau kam septisch zur Operation, nach Porro. Tod.

4) 1882. Sänger, 21jährige IIIgr. Operation neun Wochen nach dem Tode der 7 monatlichen Frucht. Diagnose vorher gestellt. Abtragung des Nebenhornes ohne vorherige Eröffnung, symperitoneale Naht des muskulären Verbindungsstranges. Versenkung mit elastischer Ligatur. Reactionslose Heilung. Frau gebar in der Folge noch zwei Mal.

Also drei Fälle aus den letzten Jahren! Nicht unwahrscheinlich daher, dass eine ganze Anzahl von vermeintlichen Tubarschwangerschaften solche eines rudimentären Uterushornes sind und waren. 
Ehe man daher, wie Litzmann-Werth, das Leben des Kindes aufgiebt, müsste doch erst entschieden werden, ob nicht statt einer tubären u. s. w. Schwangerschaft solche eines Uterushornes vorliegt. S. hält diese für wohl diagnosticirbar, namentlich mit Hülfe nachweisbarer Contractionen des Fruchthalters bei Leerbefund der ausgebildeten Uterushälfte. Bei rechtzeitigem Eingriff wäre nicht blos das Kind zu retten, sondern auch die Chance für die Mutter, von dem fatalen Nebenhorne befreit zu werden, sehr günstig, da der Stumpf desselben nie eine Höhle enthält und versenkt werden kann. - Zur Stïtze der Vermuthung Müller's von dem Vorkommen einer Ruptur des Sackes bei Extrauterinschwangerschaft erwähnt S., dass in seinem Falle eine grosse herniöse Ausbuchtung und Verdünnung des Nebenhornes bestand, welche leicht zur Ruptur führen konnte. Schliesslich erzählt S. noch einen Fall als Beitrag zur Schwierigkeit der Differentialdiagnose von Nebenhornschwangerschaft: 40jährige Igr., Porro-Operation wegen Missed labour und multiplen Myomen (im Ganzen fünf) des Uterus, wovon zwei von Doppelfaustgrösse und in Fettnecrose begriffen waren (,Amputatio uteri fibrosi gravidi“). Von dem betreffenden Arzte war Extrauterinschwangerschaft vermuthet worden. Da aber ein Myom zu fühlen war, so konnte es sich nur um einen muskulären Fruchthalter handeln, und dachte S. um so eher an Nebenhornschwangerschaft, als man, in Narkose, in eine anscheinend leere Uterushälfte hinein kam, doch war diese das Collum und der vermeintliche Fundus war eines der Myome.

Werth constatirt die im Allgemeinen übereinstimmende Auffassung der Sache. Empfiehlt Nachuntersuchung von Präparaten anatomischer Sammlungen. Müller gegenüber bemerkt er, dass unter den von Litzmann und ihm gesammelten Fällen sich 59 Frauen befauden, welche den Fruchttod uiberlebten, während von den 17 früh Operirten 15 starben. Immerhin giebt er zu, dass Berstungen, Blutungen möglich sind. Olshausen's Fall hat sich vielleicht nicht zweckmässig gehalten.

P. Müller: Seine Frage sei noch nicht beantwortet, sie lautete nach Fällen von Frauen, die nach dem Fruchttode gestorben sind. Diese müssten eine dritte statistische Reihe bilden. Wenn es ungefährlich ist, das todte Kind weiter zu tragen, brauchte man es auch nicht weiter zu schonen und könnte es auch bei vorgerückter Schwangerschaft tödten. 
334 Verhandlungen der gynäkolog. Section des VIIJ. internationalen

Werth: Wenn es so gefährlich wäre, würden nicht so viele Frauen den Fruchttod überleben. Hält es auch für wohl zulässig, die Frucht, etwa im siebenten Monate, zu tödten z. B. durch Injection eines Giftes.

Koeberlé kann einen Fall anführen, wo er der Punction eines in der Schwangerschaft vorgerückteren abdominalen Fruchtsackes Injectionen von Morphium nachschickte. Der Sack verkleinerte sich darauf. Foetus und Placenta wurden resorbirt. Vollständige Heilung.

26) Koeberlé (Strassburg): Ueber eine neue Methode der Perineorrhaphie.

Die Hauptschwierigkeit für das Gelingen der Perineoplastik in Fällen, wo auch das Rectum mit eingerissen ist, bildet das Eintreten der ersten Stuhlgänge. Dieffenbach, Freund, Hegar suchten bereits durch seitliche Schnitte oder subcutane entspannende Durchtrennungen die. Naht des neugebildeten Sphincter ani zu halten und den Faeces, Flatus ungehinderten Abfluss zu sichern. Koeberlé verfährt zu diesem Behufe in der Weise, dass er den Analring durch einen hinteren, tiefen, bis auf das Os coccygis gehenden Schnitt spaltet. Die dadurch entstandene dreieckige Wunde wird durch Verschorfung mit Chloreisen bis zur völligen Heilung der Dammwunde offen erhalten. Es ist wichtig, dass der Schnitt so hoch ins Rectum hinaufgehe, dass sicher alle Muskelfasern des Sphincter durchtrennt sind. Durch das freie Abfliessen der Faeces wird die Heilung der Dammwunde nicht gestört. Die offene Wunde wird mit Jodoform behandelt und kann man sie gewöhnlich vom 15. Tage an der Vernarbung überlassen.

27) Marcy (Boston): Ueber eine neue Methode der Perineorrhaphie.

(Manuscript von einem Vertreter Marcy's vorgelesen.) Die Anfrischung wird in der Weise vorgenommen, dass dex von der hinteren Scheidenwand abpräparirte Lappen nicht abgetragen, sondern in triangulärer Form mit der Basis nach dem Damme zu für sich vernäht wird. Die eigentliche Dammwunde wird in folgender Weise geschlossen: eine an ihrem stumpfen Ende etwa $1 \mathrm{~cm}$ breite, rechtwinkelig umgebogene Nadel besitzt ebenda eine feine Oese, durch welche eine von der anderen Seite her durchgestochene, gleichfalls rechtwinkelig umgebogene und ein Häkchen zur Aufnahme der ersten tragende zweite Nadel durchgesteckt wird. 
Werden die spitzen Enden beider Nadeln fest angezogen und dann umgebogen, so pressen die rechtwinkligen Stücke derselben die Wundränder fest aneinander. Zwischen diesen werden dann noch oberflächliche Suturen mit Thiersehnen angelegt. (Der Mechanismus ähnelt dem bekannten grausamen Knabenspielzeug aus Kirschenstengeln, der „Fliegenguillotine“. S.)

Hegar (Freiburg i/B.): Der hintere Sphincterenschnitt ist bereits von Marion Sims angegeben. Er wendet ihn gleichfalls an, doch ohne Liquor ferri auf die Wunde zu bringen. Er wird nur dann nöthig, wo auch der Anus neu zu bilden ist. Simon war der Erste, welcher die Behandlung mit constipirenden Mitteln verliess: er gab schon am dritten Tage ein Laxans. Die Schwierigkeit, welche die Entfernung der Rectalnähte bietet, umgeht Hegar jetzt dadurch, dass er von der Scheide her mit Silber sehr tief, bis nahe ans Rectum, näht und in diesem dann nur oberflächliche Catgutnähte anlegt.

Trelat (Paris) berichtet über die von ihm nach verschiedenen Methoden ausgeführten Perineoplastiken bei incompleten und completen Dammrissen. Bis vor zwei Jahren operirte er meist nach einer eigenen Methode oder derjenigen von Gaillard Thomas. Seitdem wendet er ausschliesslich die Methode von Maitte (?) in Rouen an. Anfrischung $\wedge$-förmig mit der Spitze nach der Scheide, den in der Form der Griffe einer Scheere verbreiterten Schenkeln nach der Dammfalte zu. Nur zweiseitige Naht: in der Scheide, mit Bildung einer scharfen Commissura labiorum, und am Damm. Keine Rectalnähte, kein hinterer Analschnitt! Dagegen tiefe Entspannungsnähte durch die Haut des Gesässes. -

Zambaco (Constantinopel) überreicht das Manuscript seines beabsichtigten Vortrags: „Physiologische und pathologische Betrachtungen über die Frauen des Orients" dem Secretär zur Veröffentlichung in den Comptes rendus des Congresses.

Schlussansprache von Howitz (Kopenhagen). 